\title{
Decoherence in Pre-symmetric Spaces
}

\section{Martin EdWARDS and Remo V. HügLI}

\author{
The Queen's College \\ Oxford - United Kingdom \\ martin.edwards@queens.ox.ac.uk
}

\author{
University College \\ Dublin - Ireland
}

hugli@maths.ucd.ie

Received: May 14, 2007

Accepted: October 8, 2007

\begin{abstract}
Pre-symmetric complex Banach spaces have been proposed as models for state spaces of physical systems. A structural projection on a pre-symmetric space $A_{*}$ represents an operation on the corresponding system, and has as its range a further pre-symmetric space which represents the state space of the resulting system and symmetries of the system are represented by elements of the group $\operatorname{Aut}\left(A_{*}\right)$ of linear isometries of $A_{*}$. Two structural projections $R$ and $S$ on the pre-symmetric space $A_{*}$ represent decoherent operations when their ranges are rigidly collinear. It is shown that, for decoherent elements $x$ and $y$ of $A_{*}$, there exists an involutive element $\phi_{*}$ in $\operatorname{Aut}\left(A_{*}\right)$ which conjugates the structural projections corresponding to $x$ and $y$, and conditions are found for $\phi_{*}$ to exchange $x$ and $y$. The results are used to investigate when certain subspaces of $A_{*}$ are the ranges of contractive projections and, therefore, represent systems arising from filtering operations.
\end{abstract}

Key words: $\mathrm{JBW}^{*}$-triple, pre-symmetric space, decoherence, involutive grading, exchange automorphism.

2000 Mathematics Subject Classification: Primary 46L70; Secondary 17C65, 81P15.

\section{Introduction}

This paper presents the results of further investigations into the structure and properties of pre-symmetric complex Banach spaces. A complex Banach space $A_{*}$ is said to be pre-symmetric if the open unit ball in its Banach dual space $A$ is a bounded symmetric domain. In this case $A$ possesses a natural triple product with respect to which it forms a $\mathrm{JBW}^{*}$-triple with unique predual $A_{*}$. Hence, there exists a bijection between the set of pre-symmetric spaces and the set of $\mathrm{JBW}^{*}$-triples. An 
important property of a $\mathrm{JBW}^{*}$-triple $A$ is that the group of linear isometries of $A$ to itself coincides with the group of triple automorphisms $\operatorname{Aut}(A)$ of $A$, and, because of the uniqueness of the pre-dual $A_{*}$ of $A$, each such mapping is weak*-continuous, which implies that there exists an isomorphism $\phi \mapsto \phi_{*}$ from $\operatorname{Aut}(A)$ onto the group $\operatorname{Aut}\left(A_{*}\right)$ of linear isometries of $A_{*}$. An element $\phi$ of $\operatorname{Aut}(A)$ is said to be involutive if its square is equal to the identity $\operatorname{id}_{A}$. Clearly, this property is preserved under the mapping $\phi \mapsto \phi_{*}$. There is a bijection $\phi \mapsto\left(B_{+}^{\phi}, B_{-}^{\phi}\right)$ between the family of involutive automorphisms of $A$ and that of involutive gradings of $A$, the properties of which were discussed in [17]. A further important property of a pre-symmetric space $A_{*}$ is that its image $Q A_{*}$ under a contractive projection $Q$ is also a pre-symmetric space $[29,40]$.

In some approaches to the theory of statistical physical systems, a pre-symmetric space $A_{*}$ represents the state space of the system, the linear isometries representing the symmetries of the system and the contractive projections on $A_{*}$ representing filters on the system [19-22].

A contractive projection $R$ on the pre-symmetric space $A_{*}$ is said to be structural if, for each element $x$ of $A_{*}$ such that $R x$ and $x$ have equal norm it follows that $R x$ and $x$ coincide, and, if $x$ is an element of $A_{*}$ for which, for all elements $y$ in $A_{*}$

$$
\|x+R y\|=\|x\|+\|R y\|,
$$

then $x$ lies in the kernel of $R$. Such a projection may more naturally be termed a geometric structural projection, but, in the interest of brevity, will simply be termed a structural projection. The range $R A_{*}$ of a structural projection is said to be a structural subspace of $A_{*}$. When ordered by set inclusion the family $\mathcal{I}_{*}\left(A_{*}\right)$ of structural subspaces of $A_{*}$ forms a complete lattice, as does the family $\mathcal{S}_{*}\left(A_{*}\right)$ of structural projections with respect to the order inherited from $\mathcal{I}_{*}\left(A_{*}\right)$. The adjoint $R^{*}$ of an element $R$ of $\mathcal{S}_{*}\left(A_{*}\right)$ is an element of the complete lattice $\mathcal{S}(A)$ of algebraic structural projections on the $\mathrm{JBW}^{*}$-triple $A$ the range $R^{*} A$ of which is an element of the complete lattice $\mathcal{I}(A)$ of weak* ${ }^{*}$-closed inner ideals in $A$, the structural subspace $R A_{*}$ being the predual of $R^{*} A$ [10]. The topics discussed in this paper may be viewed as properties of any one of the four order isomorphic complete lattices $\mathcal{S}_{*}\left(A_{*}\right), \mathcal{I}_{*}\left(A_{*}\right)$, $\mathcal{S}(A)$, and $\mathcal{I}(A)$. However, when considering pre-symmetric spaces as state spaces, the physical aspects are more transparent when viewed as properties of structural projections and structural subspaces of $A_{*}$.

For each element $R$ of $\mathcal{S}_{*}\left(A_{*}\right)$, there exists a unique element $R_{0}$ of $\mathcal{S}_{*}\left(A_{*}\right)$ such that

$$
R_{0} A_{*}=\left\{x \in A_{*}:\|x+y\|=\|x\|+\|y\|, \forall y \in R A_{*}\right\} .
$$

Writing

$$
R_{2}=R, \quad R_{1}=\operatorname{id}_{A_{*}}-R_{2}-R_{0},
$$

$R_{2}, R_{1}$, and $R_{0}$ are pairwise orthogonal projections, and

$$
\operatorname{id}_{A_{*}}=R_{2}+R_{1}+R_{0}
$$


is the generalized Peirce decomposition of $\operatorname{id}_{A_{*}}$ corresponding to $R$. Similarly,

$$
A_{*}=R_{2} A_{*} \oplus R_{1} A_{*} \oplus R_{0} A_{*}, \quad A=R_{2}^{*} A \oplus R_{1}^{*} A \oplus R_{0}^{*} A,
$$

are the generalized Peirce decompositions of $A_{*}$ and $A$ corresponding to the structural subspace $R_{2} A_{*}$ of $A_{*}$ and the weak*-closed inner ideal $R_{2}^{*} A$ of $A$, respectively. Observe that, although $R_{2} A_{*}$ and $R_{0} A_{*}$ are pre-symmetric spaces, with dual spaces the weak*closed inner ideals $R_{2}^{*} A$ and $R_{0}^{*} A$, since $R_{1}$ need not be contractive, $R_{1}^{*} A$ need not be a $\mathrm{JBW}^{*}$-triple and certainly need not be a subtriple of $A$. In the special case in which $R_{1}$ is contractive, the structural projection $R$ is said to be a Peirce structural projection, the same adjective being applied to the corresponding structural subspace $R A_{*}$, algebraic structural projection $R^{*}$, and inner ideal $R_{2}^{*} A$. Remarkably, this condition is equivalent to the purely algebraic condition that the Peirce spaces $R_{2}^{*} A$, $R_{1}^{*} A$, and $R_{0}^{*} A$ satisfy all of the Peirce arithmetical relations [16].

Returning to physical systems, it is clear that the two conditions that a contractive projection $R$ must satisfy in order to be structural have powerful physical motivations. In this case $R$ represents a measurement with two outcomes in which $R_{2} A_{*}$ represents the set of states with a positive outcome, $R_{0} A_{*}$ represents the set of states with a negative outcome, and $R_{1} A_{*}$ represents the information lost in the measurement. A possible interpretation of the condition that $R$ is Peirce is that that the lost information is recoverable by means of the filter represented by the contractive projection $R_{1}$

A pair $R$ and $S$ of structural projections on the pre-symmetric space $A_{*}$ is said to be compatible if, for $j$ and $k$ equal to 0,1 , and 2 , the Peirce projections $R_{j}$ and $S_{k}$ corresponding to $R$ and $S$ commute. In a recent paper, the properties of a pair $R$ and $S$ of structural projections on the pre-symmetric space $A_{*}$ which are rigidly collinear in that

$$
R_{2} A_{*} \subseteq S_{1} A_{*}, \quad S_{2} A_{*} \subseteq R_{1} A_{*},
$$

were studied [9]. Physically, such pairs may be thought to represent decoherent operations. The main results of the paper show that if $R$ and $S$ are both Peirce structural projections then the supremum $R \vee S$ in the complete lattice $\mathcal{S}_{*}\left(A_{*}\right)$ is equal to $R+S$, and that the three structural projections $R, S$, and $R+S$ are compatible, results which have clear physical interpretations.

The main purpose of this paper is to study pairs $x$ and $y$ of elements of the presymmetric space $A_{*}$ of norm one which are decoherent in the sense that the smallest structural projections $S_{x}$ and $S_{y}$ which, respectively, leave $x$ and $y$ fixed, are rigidly collinear. In this case there exists an involutive automorphism $\phi$ of $A$ the pre-adjoint $\phi_{*}$ of which conjugates the structural projections $S_{x}$ and $S_{y}$. A central result of the paper shows that $\phi_{*}$ actually maps $x$ to $y$ if and only if

$$
\|x+y\|^{2}=2 .
$$

Such pairs $x$ and $y$ of elements of $A_{*}$ of norm one are said to be strongly decoherent. 
In the theory of Banach spaces and in the study of state spaces of physical systems, much effort has been devoted to the investigation of when particular closed subspaces of a Banach space are the ranges of contractive projections. Since the range of a contractive projection on a pre-symmetric space is itself a pre-symmetric space, this problem is particularly relevant in the case of pre-symmetric spaces. The main results are used to determine under what circumstances the closed subspace of $A_{*}$ generated by a family $\left\{x_{j}: j \in \Lambda\right\}$ of elements of $A_{*}$ of norm one which are pairwise decoherent is the range of a contractive projection. Remarkably, this occurs if and only if the family is strongly decoherent.

The results of the paper depend crucially upon the detailed study of pairs of rigidly collinear tripotents in the $\mathrm{JBW}^{*}$-triple. As a consequence, some of the preliminary results given are rather more general than strictly needed to produce the result described above, but may well be of independent interest. Furthermore, the proofs of many of the results are purely algebraic, and hold in the case in which $A$ is an anisotropic Jordan*-triple.

The paper is organized as follows. Section 1 is devoted to a statement of definitions and a description of known results about $\mathrm{JBW}^{*}$-triples. In section 2 a collection of results relating to the exchange automorphisms induced by collinear pairs of tripotents are presented. Many of these have appeared elsewhere, and only those proofs that are relevant to the discussion in hand are given in detail. In section 3 , the main results of the paper are proved, and the final section is devoted to their applications.

\section{Preliminaries}

A Jordan *-algebra $A$ which is also a complex Banach space such that, for all elements $a$ and $b$ in $A,\left\|a^{*}\right\|=\|a\|,\|a \circ b\| \leq\|a\|\|b\|$ and $\left\|\left\{\begin{array}{lll}a & a & a\end{array}\right\}\right\|=\|a\|^{3}$, where

$$
\{a b c\}=a \circ\left(b^{*} \circ c\right)+\left(a \circ b^{*}\right) \circ c-b^{*} \circ(a \circ c)
$$

is the Jordan triple product on $A$, is said to be a Jordan $C^{*}$-algebra [43] or $J B^{*}$ algebra [44]. A Jordan $\mathrm{C}^{*}$-algebra which is the dual of a Banach space is said to be a Jordan $W^{*}$-algebra [8] or a $J B W^{*}$-algebra [44]. Examples of JB*-algebras are $\mathrm{C}^{*}$-algebras and examples of $\mathrm{JBW}^{*}$-algebras are $\mathrm{W}^{*}$-algebras, in both cases equipped with the Jordan product

$$
a \circ b=\frac{1}{2}(a b+b a) .
$$

The self-adjoint parts of $\mathrm{JB}^{*}$-algebras and $\mathrm{JBW}^{*}$-algebras are said to be JB-algebras and $J B W$-algebras respectively. For the properties of $\mathrm{C}^{*}$-algebras and $\mathrm{W}^{*}$-algebras the reader is referred to $[38,39]$ and for the algebraic properties of Jordan algebras to $[24,31,33,37]$.

A complex vector space $A$ equipped with a triple product $(a, b, c) \mapsto\{a b c\}$ from $A \times A \times A$ to $A$ which is symmetric and linear in the first and third variables, conjugate 
linear in the second variable and, for elements $a, b, c$, and $d$ in $A$, satisfies the identity

$$
[D(a, b), D(c, d)]=D(\{a b c\}, d)-D(c,\{d a b\}),
$$

where [ , ] denotes the commutator, and $D$ is the mapping from $A \times A$ to the algebra of linear operators on $A$ defined by

$$
D(a, b) c=\{a b c\}
$$

is said to be a Jordan*-triple. For each element $a$ in $A$, the conjugate linear mapping $Q(a)$ from $A$ to itself is defined, for each element $b$ in $A$, by

$$
Q(a) b=\left\{\begin{array}{lll}
a & b & a
\end{array}\right\} .
$$

For details about the properties of Jordan*-triples the reader is referred to [30].

A Jordan*-triple $A$ which is also a Banach space such that $D$ is continuous from $A \times A$ to the Banach algebra $B(A)$ of bounded linear operators on $A$, and, for each element $a$ in $A, D(a, a)$ is hermitian in the sense of [3, Definition 5.1], with nonnegative spectrum, and satisfies

$$
\|D(a, a)\|=\|a\|^{2},
$$

is said to be a $J B^{*}$-triple. A subspace $J$ of a $\mathrm{JB}^{*}$-triple $A$ is said to be a subtriple if $\{J J J\}$ is contained in $J$, is said to be an inner ideal if $\{J A J\}$ is contained in $J$. Every norm-closed subtriple of a $\mathrm{JB}^{*}$-triple $A$ is a $\mathrm{JB}^{*}$-triple [28], and a norm-closed subspace $J$ of $A$ is an ideal if and only if $\{J J A\}$ is contained in $J[4]$. A JB*-triple $A$ which is the dual of a Banach space $A_{*}$ is said to be a $J B W^{*}$-triple. In this case the predual $A_{*}$ of $A$ is unique and, for elements $a$ and $b$ in $A$, the operators $D(a, b)$ and $Q(a)$ are weak*-continuous. It follows that a weak*-closed subtriple $J$ of a $\mathrm{JBW}^{*}$ triple $A$ is a $\mathrm{JBW}^{*}$-triple. The second dual $A^{* *}$ of a $\mathrm{JB}^{*}$-triple $A$ is a $\mathrm{JBW}^{*}$-triple. For details of these results the reader is referred to $[1,2,6,7,23,25,28,29,41,42]$. Examples of $\mathrm{JB}^{*}$-triples are $\mathrm{JB}^{*}$-algebras, and examples of $\mathrm{JBW}^{*}$-triples are $\mathrm{JBW}^{*}$-algebras.

A pair $a$ and $b$ of elements in a $\mathrm{JBW}^{*}$-triple $A$ is said to be orthogonal when $D(a, b)$ is equal to zero. For a subset $L$ of $A$, the subset $L^{\perp}$ of $A$ consisting of all elements which are orthogonal to all elements of $L$ is a weak*-closed inner ideal in $A$ which is known as the annihilator of $L$ in $A$. For subsets $L, M$ of $A, L^{\perp} \cap L \subseteq\{0\}$, $L \subseteq L^{\perp \perp}, L \subseteq M$ implies that $M^{\perp} \subseteq L^{\perp}$, and $L^{\perp}$ and $L^{\perp \perp \perp}$ coincide.

For each non-empty subset $J$ of the $\mathrm{JBW}^{*}$-triple $A$, the kernel $\operatorname{Ker}(J)$ of $J$ is the weak*-closed subspace of elements $a$ in $A$ for which $\{J a J\}$ is equal to $\{0\}$. It follows that the annihilator $J^{\perp}$ of $J$ is contained in $\operatorname{Ker}(J)$ and that $J \cap \operatorname{Ker}(J)$ is contained in $\{0\}$. A subtriple $J$ of $A$ is said to be complemented [15] if $A$ coincides with $J \oplus \operatorname{Ker}(J)$. It can easily be seen that every complemented subtriple is a weak*-closed inner ideal. A linear projection $P$ on the $\mathrm{JBW}^{*}$-triple $A$ is said to be an algebraic structural projection [31] if, for each element $a$ in $A$,

$$
P Q(a) P=Q(P a)
$$


The main results of $[12,14,15]$ show that the range $P A$ of a structural projection $P$ is a complemented subtriple, that the kernel ker $P$ of the map $P$ coincides with $\operatorname{Ker}(P A)$, that every structural projection is contractive and weak*-continuous, and, most significantly, that every weak*-closed inner ideal is complemented.

Let $\mathcal{I}(A)$ denote the complete lattice of weak ${ }^{*}$-closed inner ideals in the $\mathrm{JBW}^{*}$ triple $A$ and let $\mathcal{S}(A)$ denote the set of structural projections on $A$. The results of [12] can be used to show that the set $\mathcal{S}(A)$ of structural projections on $A$ is a complete lattice with respect to the ordering defined, for elements $P$ and $Q$, by $P \leq Q$ if $Q P$ is equal to $P$ and the mapping $P \mapsto P A$ is an order isomorphism from $\mathcal{S}(A)$ onto the complete lattice $\mathcal{I}(A)$ of weak*-closed inner ideals in $A$. For an element $P$ in $\mathcal{S}(A)$, the pre-adjoint $P_{*}$ is an element of the complete lattice $\mathcal{S}_{*}\left(A_{*}\right)$ of structural projections on the pre-symmetric space $A_{*}$, and the mappings $P \mapsto P_{*}$ and $P \mapsto P_{*} A_{*}$ are order isomorphisms from $\mathcal{S}(A)$ onto the complete lattices $\mathcal{S}_{*}\left(A_{*}\right)$ and $\mathcal{I}_{*}\left(A_{*}\right)$, respectively [10].

For each element $J$ of $\mathcal{I}(A)$, the annihilator $J^{\perp}$ also lies in $\mathcal{I}(A)$ and $A$ enjoys the generalized Peirce decomposition

$$
A=J_{0} \oplus J_{1} \oplus J_{2}
$$

where

$$
J_{0}=J^{\perp}, \quad J_{2}=J, \quad J_{1}=\operatorname{Ker}(J) \cap \operatorname{Ker}\left(J^{\perp}\right) .
$$

The structural projections onto $J$ and $J^{\perp}$ are denoted by $P_{2}(J)$ and $P_{0}(J)$, respectively, and the projection $\operatorname{id}_{A}-P_{2}(J)-P_{0}(J)$ onto $J_{1}$ is denoted by $P_{1}(J)$. Furthermore,

$$
\left\{A J_{0} J_{2}\right\}=\{0\}, \quad\left\{A J_{2} J_{0}\right\}=\{0\} .
$$

and, for $j, k$, and $l$ equal to 0,1 , or 2 , the Peirce arithmetical relations,

$$
\left\{J_{j} J_{k} J_{l}\right\} \subseteq J_{j+l-k},
$$

when $j+l-k$ is equal to 0,1 , or 2 , and

$$
\left\{J_{j} J_{k} J_{l}\right\}=\{0\},
$$

otherwise, hold, except in the cases when $(j, k, l)$ is equal to $(0,1,1),(1,1,0),(1,0,1)$, $(2,1,1),(1,1,2),(1,2,1)$, or $(1,1,1)$. The relations $(9),(10)$ hold in all cases if and only if $P_{1}(J)$ is contractive in which case $J$ is said to be a Peirce inner ideal. The word Peirce is also used to describe the corresponding algebraic structural projection on $A$, structural projection on $A_{*}$, and structural subspace of $A_{*}$. A pair $J$ and $K$ of elements of $\mathcal{I}(A)$ is said to be compatible if, for $j$ and $k$ equal to 0,1 , or 2 ,

$$
\left[P_{j}(J), P_{k}(K)\right]=0,
$$

or, equivalently, if

$$
A=\bigoplus_{j, k=0,1,2} J_{j} \cap K_{k}
$$


[11]. The pair $J$ and $K$ is said to be rigidly collinear if $J_{2}$ is contained in $K_{1}$ and $K_{2}$ is contained in $J_{1}$. If $J$ and $K$ are Peirce and form a rigidly collinear pair then it can be shown that the subspace $J+K$ of $A$ is a weak*-closed inner ideal in $A$, the Peirce spaces corresponding to $J+K$ are given by

$$
\begin{aligned}
& (J+K)_{2}=J+K, \\
& (J+K)_{1}=J_{1} \cap K_{1} \oplus J_{1} \cap K_{0} \oplus J_{0} \cap K_{1}, \\
& (J+K)_{0}=J_{0} \cap K_{0},
\end{aligned}
$$

with corresponding Peirce projections

$$
\begin{aligned}
& P_{2}(J+K)=P_{2}(J)+P_{2}(K), \\
& P_{1}(J+K)=P_{1}(J) P_{1}(K)+P_{1}(J) P_{0}(K)+P_{0}(J) P_{1}(K), \\
& P_{0}(J+K)=P_{0}(J) P_{0}(K),
\end{aligned}
$$

and the weak ${ }^{*}$-closed inner ideals $J, K$, and $J+K$ form a pairwise compatible family $[9]$.

Let $A$ be a $\mathrm{JBW}^{*}$-triple with predual $A_{*}$ and let $\operatorname{Aut}(A)$ denote the group of linear isometries from $A$ to itself. This coincides with the group of triple automorphisms of $A$. Each element $\phi$ of $\operatorname{Aut}(A)$ is weak*-continuous and, if $\phi_{*}$ denotes the pre-adjoint of $\phi$ then the mapping $\phi \mapsto \phi_{*}$ is an isomorphism from the group $\operatorname{Aut}(A)$ onto the $\operatorname{group} \operatorname{Aut}\left(A_{*}\right)$ of linear isometries of $A$.

Recall that a pair $\left(B_{+}, B_{-}\right)$of weak*-closed subtriples of a $\mathrm{JBW}^{*}$-triple $A$ is said to be an involutive grading of $A$ if

$$
\begin{gathered}
A=B_{+} \oplus B_{-}, \\
\left\{B_{+} B_{-} B_{+}\right\} \subseteq B_{-}, \quad\left\{B_{-} B_{+} B_{-}\right\} \subseteq B_{+}, \\
\left\{B_{+} B_{+} B_{-}\right\} \subseteq B_{-}, \quad\left\{B_{-} B_{-} B_{+}\right\} \subseteq B_{+} .
\end{gathered}
$$

Observe that, by symmetry, if $\left(B_{+}, B_{-}\right)$is an involutive grading then so also is $\left(B_{-}, B_{+}\right)$which, in this case, is said to be the opposite grading. An element $\phi$ of the group $\operatorname{Aut}(A)$ of triple automorphisms of $A$ which satisfies the condition that $\phi^{2}$ coincides with the identity $\mathrm{id}_{A}$, is said to be an involutive automorphism of $A$. Notice that if $\phi$ is an involutive automorphism of $A$ then so also is $-\phi$. Let $A$ be a $\mathrm{JBW}^{*}$-triple, let $\phi$ be an involutive automorphism of $A$, and let

$$
B_{+}^{\phi}=\{a \in A: \phi a=a\}, \quad B_{-}^{\phi}=\{a \in A: \phi a=-a\} .
$$

Then, $\left(B_{+}^{\phi}, B_{-}^{\phi}\right)$ is an involutive grading and the mapping $\phi \mapsto\left(B_{+}^{\phi}, B_{-}^{\phi}\right)$ is a bijection from the set of involutive automorphisms of $A$ onto the set of involutive gradings of $A$, such that $\left(B_{+}^{-\phi}, B_{-}^{-\phi}\right)$ coincides with $\left(B_{-}^{\phi}, B_{+}^{\phi}\right)$. Furthermore, the pre-adjoint $\phi_{*}$ of an involutive automorphism gives rise to an involutive grading $\left(B_{*+}^{\phi_{*}}, B_{*-}^{\phi_{*}}\right)$ of the predual $A_{*}$ of $A$ in which $B_{*+}^{\phi_{*}}$ and $B_{*-}^{\phi_{*}}$ may be identified with the preduals of $B_{+}^{\phi}$ 
and $B_{-}^{\phi}$, respectively. It is clear that, for an involutive automorphism $\phi$ of the JBW*triple $A$, with corresponding involutive grading $\left(B_{+}^{\phi}, B_{-}^{\phi}\right)$, the weak*-continuous linear mapping $T_{\phi}$, defined by

$$
T_{\phi}=\frac{1}{2}\left(\mathrm{id}_{A}+\phi\right),
$$

is the linear projection onto the weak ${ }^{*}$-closed subtriple $B_{+}^{\phi}$ and $T_{-\phi}$ is the linear projection onto the weak*-closed subtriple $B_{-}^{\phi}$. Clearly, the projections $T_{\phi}$ and $T_{-\phi}$ are orthogonal. Similarly, the orthogonal projections $T_{\phi_{*}}$ and $T_{-\phi_{*}}$ map onto $B_{*+}^{\phi_{*}}$ and $B_{*-}^{\phi_{*}}$, respectively. The reader is referred to $[17,36]$ for details.

\section{Exchange automorphisms}

An element $u$ in a $\mathrm{JBW}^{*}$-triple $A$ is said to be a tripotent if $\{u u u\}$ is equal to $u$. The set of tripotents in $A$ is denoted by $\mathcal{U}(A)$. Observe that, by (4), non-zero tripotents are of norm one. For each tripotent $u$ in $A$, the weak*-continuous linear operators $P_{0}(u), P_{1}(u)$ and $P_{2}(u)$, defined using $(2),(3)$ by

$$
\begin{gathered}
P_{0}(u)=\operatorname{id}_{A}-2 D(u, u)+Q(u)^{2}, \quad P_{1}(u)=2\left(D(u, u)-Q(u)^{2}\right), \\
P_{2}(u)=Q(u)^{2},
\end{gathered}
$$

are mutually orthogonal projection operators on $A$ with $\operatorname{sum~id}_{A}$. For $j$ equal to 0,1 , or 2 , the range of $P_{j}(u)$ is the weak ${ }^{*}$-closed eigenspace $A_{j}(u)$ of $D(u, u)$ corresponding to the eigenvalue $\frac{1}{2} j$ and

$$
A=A_{0}(u) \oplus A_{1}(u) \oplus A_{2}(u)
$$

is the Peirce decomposition of $A$ relative to $u$. Moreover, $A_{2}(u)$ is a Peirce weak*closed inner ideal in $A$, and, consequently, the Peirce arithmetical relations (8)-(10) hold. Observe that when endowed with the product $(a, b) \mapsto a \circ_{u} b=\{a \quad u b\}$ and the involution $a \mapsto a^{\dagger u}=\left\{\begin{array}{lll}u & a & u\end{array}\right\}$ the Peirce space $A_{2}(u)$ forms a $\mathrm{JBW}^{*}$-algebra with unit $u$.

Elements $u$ and $v$ in $\mathcal{U}(A)$ are said to be compatible if the weak*-closed inner ideals $A_{2}(u)$ and $A_{2}(v)$ are compatible. Observe that $u$ and $v$ are compatible if $u$ lies in a Peirce $j$-space of $v$ [32]. For compatible tripotents $u$ and $v$, and, for $j$ and $k$ equal to 0 , 1 , or 2 , denote by $A_{j k}(u, v)$ the intersection of $A_{j}(u)$ and $A_{k}(v)$. The corresponding orthogonal decomposition of $A$ is said to be the joint Peirce decomposition of $A$ corresponding to $u$ and $v$. Two tripotents $u$ and $v$ are said to be orthogonal if one of the following equivalent conditions hold: $D(u, v)=0 ; D(v, u)=0 ;\{u u v\}=0$; $\{v v u\}=0 ; v$ lies in $A_{0}(u) ; u$ lies in $A_{0}(v)$. Observe that there exists a partial order on $\mathcal{U}(A)$ defined, for elements $u$ and $v$ in $\mathcal{U}(A)$, by $u \leq v$ if $\{u v u\}$ is equal to $u$, or, equivalently if $v-u$ is a tripotent orthogonal to $u$. The set $\tilde{\mathcal{U}}(A)$ consisting of $\mathcal{U}(A)$ with a largest element adjoined forms a complete lattice. Two non-zero tripotents $u$ and $v$ are said to be collinear if $u$ lies in $A_{1}(v)$ and $v$ lies in $A_{1}(u)$ and rigidly collinear 
if $A_{2}(u)$ and $A_{2}(v)$ form a pair of rigidly collinear weak* ${ }^{*}$-closed inner ideals. For more details the reader is referred to $[30,33,34]$.

Let $A$ be a $\mathrm{JBW}^{*}$-triple and let $u$ and $v$ be a pair of collinear elements of the partially ordered set $\mathcal{U}(A)$ of tripotents in $A$. Observe that, because of the symmetry of the collinearity relation any result that holds for one element of a collinear pair also holds for the other. A calculation using (6)-(10) shows that the mapping $\phi_{u, v}$ defined by

$$
\phi_{u, v}=\operatorname{id}_{A}-2 D(u+v, u+v)+Q(u+v)^{2},
$$

is an involutive automorphism of $A$ such that

$$
\phi_{u, v} u=v, \quad \phi_{u, v} v=u .
$$

The involutive automorphism $\phi_{u, v}$ is known as the exchange automorphism corresponding to the collinear pair $u$ and $v$. For an arbitrary element $w$ of $\mathcal{U}(A)$, the involutive automorphism

$$
\psi_{w}=P_{2}(w)-P_{1}(w)+P_{0}(w)
$$

is known as the Peirce reflection corresponding to $w$. A further calculation using (9), (10) shows that $2^{-\frac{1}{2}}(u+v)$ is a non-zero element of $\mathcal{U}(A)$ such that

$$
\phi_{u, v}=\psi_{2^{-\frac{1}{2}}(u+v)} .
$$

Similarly, for all elements $\alpha$ and $\beta$ of $\mathbb{C}$ for which

$$
|\alpha|^{2}+|\beta|^{2}=1,
$$

$\alpha u+\beta v$ is a non-zero element of $\mathcal{U}(A)$. Since non-zero tripotents are of norm one it follows that the restriction of the norm in $A$ to the subspace $\mathbb{C} u \oplus \mathbb{C} v$ is Hilbertian, with $u$ and $v$ perpendicular unit vectors in this subspace. Observe that the involutive grading $\left(B_{+}^{\phi_{u, v}}, B_{-}^{\phi_{u, v}}\right)$ corresponding to the involutive automorphism $\phi_{u, v}$ is given by

$$
B_{+}^{\phi_{u, v}}=A_{2}\left(2^{-\frac{1}{2}}(u+v)\right) \oplus A_{0}\left(2^{-\frac{1}{2}}(u+v)\right), \quad B_{-}^{\phi_{u, v}}=A_{1}\left(2^{-\frac{1}{2}}(u+v)\right),
$$

from which, using (11), it can be seen that

$$
\begin{aligned}
T_{\phi_{u, v}} & =\frac{1}{2}\left(\operatorname{id}_{A}+\phi_{u, v}\right)=P_{2}\left(2^{-\frac{1}{2}}(u+v)\right)+P_{0}\left(2^{-\frac{1}{2}}(u+v)\right), \\
T_{-\phi_{u, v}}=\frac{1}{2}\left(\operatorname{id}_{A}-\phi_{u, v}\right) & =P_{1}\left(2^{-\frac{1}{2}}(u+v)\right) .
\end{aligned}
$$

Furthermore, since $\phi_{u, v}$ is an automorphism, by (12),

$$
\phi_{u, v} D(u, u)=D(v, v) \phi_{u, v}, \quad \phi_{u, v} Q(u)=Q(v) \phi_{u, v},
$$

from which it follows that, for $j$ equal to 0,1 , or 2 ,

$$
\phi_{u, v} P_{j}(u)=P_{j}(v) \phi_{u, v} .
$$


Let $\left(\phi_{u, v}\right)_{*}$ be the involutive linear isometry of the predual $A_{*}$ of $A$ which is the preadjoint of the involutive automorphism $\phi_{u, v}$. Then, it is clear that the corresponding involutive grading $\left(B_{*+}^{\left(\phi_{u, v}\right)_{*}}, B_{*-}^{\left(\phi_{u, v}\right)_{*}}\right)$ of $A_{*}$ satisfies

$$
\begin{aligned}
& B_{*+}^{\left(\phi_{u, v}\right)_{*}}=B_{+*}^{\phi_{u, v}}=A_{2}\left(2^{-\frac{1}{2}}(u+v)\right)_{*} \oplus A_{0}\left(2^{-\frac{1}{2}}(u+v)\right)_{*}, \\
& B_{*-}^{\left(\phi_{u, v}\right)_{*}}=B_{-*}^{\phi_{u, v}}=A_{1}\left(2^{-\frac{1}{2}}(u+v)\right)_{*} .
\end{aligned}
$$

Moreover

$$
\begin{aligned}
T_{\left(\phi_{u, v}\right)_{*}} & =\frac{1}{2}\left(\operatorname{id}_{A_{*}}+\left(\phi_{u, v}\right)_{*}\right)=P_{2}\left(2^{-\frac{1}{2}}(u+v)\right)_{*}+P_{0}\left(2^{-\frac{1}{2}}(u+v)\right)_{*}, \\
T_{-\left(\phi_{u, v}\right)_{*}} & =\frac{1}{2}\left(\operatorname{id}_{A_{*}}-\left(\phi_{u, v}\right)_{*}\right)=P_{1}\left(2^{-\frac{1}{2}}(u+v)\right)_{*} .
\end{aligned}
$$

The action of $\phi_{u, v}$ on the joint Peirce spaces of $u$ and $v$ is given in the following result, the proof of which can be found in [34, Theorem 1.1].

Lemma 2.1. Let $A$ be a $J B W^{*}$-triple, let $u$ and $v$ be a pair of collinear tripotents in $A$ with corresponding Peirce spaces $A_{0}(u), A_{1}(u)$, and $A_{2}(u)$ and $A_{0}(v), A_{1}(v)$, and $A_{2}(v)$, respectively, let $\phi_{u, v}$ be the exchange automorphism associated with $u$ and $v$ and, for $j$ and $k$ equal to 0,1 , or 2 , let

$$
A_{j, k}(u, v)=A_{j}(u) \cap A_{k}(v) .
$$

Then, the following results hold:

(i) $\phi_{u, v} A_{22}(u, v)=A_{22}(u, v)$ and $\left.\phi_{u, v}\right|_{A_{22}(u, v)}=\operatorname{id}_{A_{22}(u, v)}$,

(ii) $\phi_{u, v} A_{21}(u, v)=A_{12}(u, v)$ and $\left.\phi_{u, v}\right|_{A_{21}(u, v)}=\left.2 D(v, u)\right|_{A_{21}(u, v)}$,

(iii) $\phi_{u, v} A_{20}(u, v)=A_{02}(u, v)$ and $\left.\phi_{u, v}\right|_{A_{20}(u, v)}=\left.Q(v) Q(u)\right|_{A_{20}(u, v)}$,

(iv) $\phi_{u, v} A_{11}(u, v)=A_{11}(u, v)$ and $\left.\phi_{u, v}\right|_{A_{11}(u, v)}=\left.\left(4 D(u, v) D(v, u)-\mathrm{id}_{A}\right)\right|_{A_{11}(u, v)}$,

(v) $\phi_{u, v} A_{10}(u, v)=A_{01}(u, v)$ and $\left.\phi_{u, v}\right|_{A_{10}(u, v)}=-\left.2 D(v, u)\right|_{A_{10}(u, v)}$,

(vi) $\phi_{u, v} A_{00}(u, v)=A_{00}(u, v)$ and $\left.\phi_{u, v}\right|_{A_{00}(u, v)}=\operatorname{id}_{A_{00}(u, v)}$.

The proof of the following result may be found in [32, Lemma 3.4].

Lemma 2.2. Let $A$ be a JBW*-triple, let $u$ and $v$ be a pair of collinear tripotents in $A$ with corresponding Peirce spaces $A_{0}(u), A_{1}(u)$, and $A_{2}(u)$ and $A_{0}(v), A_{1}(v)$, and $A_{2}(v)$, respectively. Then, the following are equivalent.

(i) $u$ and $v$ form a rigidly collinear pair,

(ii) $A_{2}(u) \subseteq A_{1}(v)$,

(iii) $A_{2}(v) \subseteq A_{1}(u)$,

(iv) $A_{2}(u) \cap A_{2}(v)=\{0\}$. 
A combination of Lemmas 2.1 and 2.2 yields the following result.

Lemma 2.3. Let $A$ be a JBW*-triple, let $u$ and $v$ be a pair of rigidly collinear tripotents in $A$ with corresponding Peirce spaces $A_{0}(u), A_{1}(u)$, and $A_{2}(u)$ and $A_{0}(v)$, $A_{1}(v)$, and $A_{2}(v)$, respectively, let $\phi_{u, v}$ be the exchange automorphism associated with $u$ and $v$ and, for $j$ and $k$ equal to 0,1 , or 2 , let

$$
A_{j, k}(u, v)=A_{j}(u) \cap A_{k}(v) .
$$

Then, the following results hold:

(i) $\phi_{u, v} A_{2}(u)=A_{2}(v)$ and $\left.\phi_{u, v}\right|_{A_{2}(u)}=\left.2 D(v, u)\right|_{A_{2}(u)}$,

(ii) $\phi_{u, v} A_{11}(u, v)=A_{11}(u, v)$ and $\left.\phi_{u, v}\right|_{A_{11}(u, v)}=\left.\left(4 D(u, v) D(v, u)-\mathrm{id}_{A}\right)\right|_{A_{11}(u, v)}$,

(iii) $\phi_{u, v} A_{10}(u, v)=A_{01}(u, v)$ and $\left.\phi_{u, v}\right|_{A_{10}(u, v)}=-\left.2 D(v, u)\right|_{A_{10}(u, v)}$,

(iv) $\phi_{u, v} A_{00}(u, v)=A_{00}(u, v)$ and $\left.\phi_{u, v}\right|_{A_{00}(u, v)}=\mathrm{id}_{A_{00}(u, v)}$.

The following result is an immediate consequence of [9, Theorem 3.5].

Lemma 2.4. Let $A$ be a JBW*-triple, let $u$ and $v$ be a pair of rigidly collinear tripotents in $A$ with corresponding Peirce spaces $A_{0}(u), A_{1}(u)$, and $A_{2}(u)$ and $A_{0}(v)$, $A_{1}(v)$, and $A_{2}(v)$, and Peirce projections $P_{0}(u), P_{1}(u)$, and $P_{2}(u)$ and $P_{0}(v), P_{1}(v)$, and $P_{2}(v)$, respectively, and, for $j$ and $k$ equal to 0 , 1 , or 2 , let

$$
A_{j, k}(u, v)=A_{j}(u) \cap A_{k}(v) .
$$

Then, the following results hold.

(i) $A_{2}(u) \oplus A_{2}(v)$ is a weak ${ }^{*}$-closed inner ideal in A with corresponding Peirce decomposition

$$
\begin{aligned}
A & =\left(A_{2}(u) \oplus A_{2}(v)\right)_{2} \oplus\left(A_{2}(u) \oplus A_{2}(v)\right)_{1} \oplus\left(A_{2}(u) \oplus A_{2}(v)\right)_{0} \\
& =\left(A_{2}(u) \oplus A_{2}(v)\right) \oplus\left(A_{11}(u, v) \oplus A_{1,0}(u, v) \oplus A_{0,1}(u, v)\right) \oplus A_{00}(u, v) .
\end{aligned}
$$

(ii) The corresponding Peirce projections are given by

$$
\begin{aligned}
& P_{2}\left(A_{2}(u) \oplus A_{2}(v)\right)=P_{2}(u)+P_{2}(v), \\
& P_{1}\left(A_{2}(u) \oplus A_{2}(v)\right)=P_{1}(u) P_{1}(v)+P_{1}(u) P_{0}(v)+P_{0}(u) P_{1}(v), \\
& P_{0}\left(A_{2}(u) \oplus A_{2}(v)\right)=P_{0}(u) P_{0}(v) .
\end{aligned}
$$

(iii) The weak* ${ }^{*}$ closed inner ideals $A_{2}(u), A_{2}(v)$, and $A_{2}(u) \oplus A_{2}(v)$ are pairwise compatible. 
Recall that it need not be the case that the weak* ${ }^{*}$ closed inner ideal $A_{2}(u) \oplus A_{2}(v)$ that appears in the previous lemma, is Peirce. Indeed, it is easy to find examples for which it is not. It is not true in general that the tripotent $2^{-\frac{1}{2}}(u+v)$ is compatible with $u$ and $v$. However, it is possible to make some remarks about the relationship between the Peirce spaces corresponding to $2^{-\frac{1}{2}}(u+v)$ and those corresponding to $u$ and $v$.

Lemma 2.5. Under the conditions of Lemma 2.3, let $A_{0}\left(2^{-\frac{1}{2}}(u+v)\right), A_{1}\left(2^{-\frac{1}{2}}(u+v)\right)$, and $A_{2}\left(2^{-\frac{1}{2}}(u+v)\right)$ and $P_{0}\left(2^{-\frac{1}{2}}(u+v)\right), P_{1}\left(2^{-\frac{1}{2}}(u+v)\right)$, and $P_{2}\left(2^{-\frac{1}{2}}(u+v)\right)$ be the Peirce spaces and Peirce projections corresponding to the tripotent $2^{-\frac{1}{2}}(u+v)$ in $A$. Then, the following results hold.

(i) $A_{2}\left(2^{-\frac{1}{2}}(u+v)\right) \subseteq A_{2}(u) \oplus A_{2}(v)$,

(ii) $A_{0}\left(2^{-\frac{1}{2}}(u+v)\right) \cap\left(A_{2}(u) \oplus A_{2}(v)\right)=\{0\}$,

(iii) $2^{-\frac{1}{2}}(u+v)$ is a maximal tripotent in the $J B W^{*}$-triple $A_{2}(u) \oplus A_{2}(v)$,

(iv) $\left(\operatorname{id}_{A}+\phi_{u, v}\right) A_{2}(u) \subseteq A_{2}\left(2^{-\frac{1}{2}}(u+v)\right)$,

(v) $P_{0}\left(2^{-\frac{1}{2}}(u+v)\right) P_{2}(u)=0$,

(vi) $P_{2}\left(2^{-\frac{1}{2}}(u+v)\right) P_{2}(u)=\frac{1}{2}\left(\operatorname{id}_{A}+\phi_{u, v}\right) P_{2}(u)$,

(vii) $P_{2}(u) P_{2}\left(2^{-\frac{1}{2}}(u+v)\right) P_{2}(u)=\frac{1}{2} P_{2}(u)$,

(viii) $P_{2}\left(2^{-\frac{1}{2}}(u+v)\right) P_{2}(u) P_{2}\left(2^{-\frac{1}{2}}(u+v)\right)=\frac{1}{2} P_{2}\left(2^{-\frac{1}{2}}(u+v)\right)$.

Proof. (i) It can be seen that the tripotent $2^{-\frac{1}{2}}(u+v)$ lies in $A_{2}(u) \oplus A_{2}(v)$ which, by Lemma 2.4 , is a weak* ${ }^{*}$-closed inner ideal in $A$. Since $A_{2}\left(2^{-\frac{1}{2}}(u+v)\right)$ is the smallest weak ${ }^{*}$-closed inner ideal containing $2^{-\frac{1}{2}}(u+v)$, the result follows.

(ii) Let $a$ be an element of $A_{0}\left(2^{-\frac{1}{2}}(u+v)\right) \cap\left(A_{2}(u) \oplus A_{2}(v)\right)$ and let $b$ and $c$ be the unique elements of $A_{2}(u)$ and $A_{2}(v)$, respectively, such that

$$
a=b+c .
$$

Then, using (8)-(10) and Lemma 2.3 (i),

$$
\begin{aligned}
& 0=2\left\{2^{-\frac{1}{2}}(u+v) 2^{-\frac{1}{2}}(u+v) a\right\}=\left\{\begin{array}{lll}
u & a
\end{array}\right\}+\left\{\begin{array}{lll}
v & a
\end{array}\right\}+\left\{\begin{array}{lll}
v & u & a
\end{array}\right\}+\left\{\begin{array}{lll}
v & v & a
\end{array}\right\} \\
& =\{u u b\}+\{u v b\}+\{v u b\}+\{v v b\} \\
& +\{u u c\}+\{u v c\}+\{v u c\}+\{v v c\} \\
& =b+0+D(v, u) b+\frac{1}{2} b+\frac{1}{2} c+D(u, v) c+0+c \\
& =\left(\frac{3}{2} b+\frac{1}{2} \phi_{u, v} c\right)+\left(\frac{3}{2} c+\frac{1}{2} \phi_{u, v} c\right) \text {. }
\end{aligned}
$$

It follows that

$$
3 b+\phi_{u, v} c=-3 c-\phi_{u, v} b \in A_{2}(u) \cap A_{2}(v)=\{0\},
$$


by Lemma 2.2. Hence,

$$
9 b=-3 \phi_{u, v} c=\phi_{u, v}^{2} b=b,
$$

and $b$ and, similarly, $c$, is equal to zero, as required.

(iii) This is immediate from the previous two results.

(iv) By Lemma 2.3 (i) it can be seen that

$$
\left(\operatorname{id}_{A}+\phi_{u, v}\right) A_{2}(u) \subseteq A_{2}(u) \oplus A_{2}(v) .
$$

Let $a$ be an element of $A_{2}(u)$. Then, by (14), the element $\left(\operatorname{id}_{A}+\phi_{u, v}\right) a$ lies in the weak* ${ }^{*}$-closed subtriple $B_{+}^{\phi_{u, v}}$ which, by (13), coincides with $A_{2}\left(2^{-\frac{1}{2}}(u+v)\right) \oplus$ $A_{0}\left(2^{-\frac{1}{2}}(u+v)\right)$. Therefore,

$$
\left(\operatorname{id}_{A}+\phi_{u, v}\right) a=P_{2}\left(2^{-\frac{1}{2}}(u+v)\right)\left(\operatorname{id}_{A}+\phi_{u, v}\right) a+P_{0}\left(2^{-\frac{1}{2}}(u+v)\right)\left(\operatorname{id}_{A}+\phi_{u, v}\right) a,
$$

From above, it follows that $P_{0}\left(2^{-\frac{1}{2}}(u+v)\right)\left(\mathrm{id}_{A}+\phi_{u, v}\right) a$ is the difference of two elements of $A_{2}(u) \oplus A_{2}(v)$ which simultaneously lies in $A_{0}\left(2^{-\frac{1}{2}}(u+v)\right)$. By (ii) it is therefore equal to zero and

$$
\left(\operatorname{id}_{A}+\phi_{u, v}\right) a=P_{2}\left(2^{-\frac{1}{2}}(u+v)\right)\left(\operatorname{id}_{A}+\phi_{u, v}\right) a,
$$

which lies in $A_{2}\left(2^{-\frac{1}{2}}(u+v)\right)$, as required.

(v) Using (14) and (iv) above, for each element $a$ in $A$,

$$
2\left(P_{2}\left(2^{-\frac{1}{2}}(u+v)\right)+P_{0}\left(2^{-\frac{1}{2}}(u+v)\right)\right) P_{2}(u) a=\left(\operatorname{id}_{A}+\phi_{u, v}\right) P_{2}(u) a,
$$

which lies in $A_{2}\left(2^{-\frac{1}{2}}(u+v)\right)$. Hence, the element

$$
\left.P_{0}\left(2^{-\frac{1}{2}}(u+v)\right)\right) P_{2}(u) a \in A_{2}\left(2^{-\frac{1}{2}}(u+v)\right) \cap A_{0}\left(2^{-\frac{1}{2}}(u+v)\right)=\{0\},
$$

as required.

(vi) Using (14) and (v) above,

$$
\begin{aligned}
\frac{1}{2}\left(\operatorname{id}_{A}+\phi_{u, v}\right) P_{2}(u) & =P_{2}\left(2^{-\frac{1}{2}}(u+v)\right) P_{2}(u)+P_{0}\left(2^{-\frac{1}{2}}(u+v)\right) P_{2}(u) \\
& =P_{2}\left(2^{-\frac{1}{2}}(u+v)\right) P_{2}(u),
\end{aligned}
$$

as required.

(vii) By (15), Lemma 2.2, the compatibility of $u$ and $v$, and (v), (vi) above,

$$
\begin{aligned}
P_{2}(u) P_{2}\left(2^{-\frac{1}{2}}(u+v)\right) P_{2}(u) & =P_{2}(u)\left(\frac{1}{2}\left(\operatorname{id}_{A}+\phi_{u, v}\right)-P_{0}\left(2^{-\frac{1}{2}}(u+v)\right)\right) P_{2}(u) \\
& =P_{2}(u)\left(\frac{1}{2}\left(\operatorname{id}_{A}+\phi_{u, v}\right)\right) P_{2}(u) \\
& =\frac{1}{2} P_{2}(u)+\frac{1}{2} P_{2}(u) P_{2}(v) \phi_{u, v}=\frac{1}{2} P_{2}(u),
\end{aligned}
$$


as required.

(viii) By (13), (14), (15), and (i) and (vi) above,

$$
\begin{aligned}
P_{2}\left(2^{-\frac{1}{2}}(u+v)\right) P_{2}(u) P_{2}\left(2^{-\frac{1}{2}}(u+v)\right)= & \frac{1}{2}\left(\operatorname{id}_{A}+\phi_{u, v}\right) P_{2}(u) P_{2}\left(2^{-\frac{1}{2}}(u+v)\right) \\
= & \frac{1}{2} P_{2}(u) P_{2}\left(2^{-\frac{1}{2}}(u+v)\right) \\
& +\frac{1}{2} P_{2}(v) \phi_{u, v} P_{2}\left(2^{-\frac{1}{2}}(u+v)\right) \\
= & \frac{1}{2}\left(P_{2}(u)+P_{2}(v)\right) P_{2}\left(2^{-\frac{1}{2}}(u+v)\right) \\
= & \frac{1}{2} P_{2}\left(2^{-\frac{1}{2}}(u+v)\right)
\end{aligned}
$$

as required.

It is now possible to prove the main result of this section which shows that, for a pair of rigidly collinear tripotents $u$ and $v$ in a $\mathrm{JBW}^{*}$-triple $A$, the involutive automorphism $\phi_{u, v}$ from $A_{2}(u)$ to $A_{2}(v)$ may, using projections, be factored in a transparent manner through $A_{2}\left(2^{-\frac{1}{2}}(u+v)\right)$.

Theorem 2.6. Let $A$ be a JBW*-triple, let $u$ and $v$ be a pair of rigidly collinear tripotents in $A$, let $\phi_{u, v}$ be the exchange automorphism associated with $u$ and $v$ and, for $j$ equal to 0,1 , or 2 , let $A_{j}(u), A_{j}(v)$, and $A_{j}\left(2^{-\frac{1}{2}}(u+v)\right)$ and $P_{j}(u), P_{j}(v)$, and $P_{j}\left(2^{-\frac{1}{2}}(u+v)\right)$ be the Peirce spaces and Peirce projections corresponding to the tripotents $u, v$, and $2^{-\frac{1}{2}}(u+v)$. Then, the following results hold.

(i) The mappings $\left.2^{\frac{1}{2}} P_{2}\left(2^{-\frac{1}{2}}(u+v)\right)\right|_{A_{2}(u)}: A_{2}(u) \rightarrow A_{2}\left(2^{-\frac{1}{2}}(u+v)\right)$ and $\left.2^{\frac{1}{2}} P_{2}(u)\right|_{A_{2}\left(2^{-\frac{1}{2}}(u+v)\right)}: A_{2}\left(2^{-\frac{1}{2}}(u+v)\right) \rightarrow A_{2}(u)$ are isometric triple isomorphisms onto the $J B W^{*}$-triples $A_{2}\left(2^{-\frac{1}{2}}(u+v)\right)$ and $A_{2}(u)$, respectively, and are inverses of each other.

(ii) The mapping $\left.2^{\frac{1}{2}} P_{2}\left(2^{-\frac{1}{2}}(u+v)\right)\right|_{A_{2}(u)}$ coincides with the mapping $\left.2^{-\frac{1}{2}}\left(\operatorname{id}_{A}+\phi_{u, v}\right)\right|_{A_{2}(u)}$.

(iii) The composition of the mappings $\left.2^{\frac{1}{2}} P_{2}\left(2^{-\frac{1}{2}}(u+v)\right)\right|_{A_{2}(u)}$ and $\left.2^{\frac{1}{2}} P_{2}(v)\right|_{A_{2}\left(2^{-\frac{1}{2}}(u+v)\right)}$ coincides with the mapping $\left.\phi_{u, v}\right|_{A_{2}(u)}$.

Proof. Let $a$ be an element of $A_{2}(u)$. Then, by Lemma 2.5 (vii),

$$
2^{\frac{1}{2}} P_{2}(u) 2^{\frac{1}{2}} P_{2}\left(2^{-\frac{1}{2}}(u+v)\right) a=2 P_{2}(u) P_{2}\left(2^{-\frac{1}{2}}(u+v)\right) P_{2}(u) a=P_{2}(u) a=a .
$$

For an element $a$ in $A_{2}\left(2^{-\frac{1}{2}}(u+v)\right)$, by Lemma 2.5 (viii),

$$
\begin{aligned}
2^{\frac{1}{2}} P_{2}\left(2^{-\frac{1}{2}}(u+v)\right) 2^{\frac{1}{2}} P_{2}(u) a & =2 P_{2}\left(2^{-\frac{1}{2}}(u+v)\right) P_{2}(u) 2^{\frac{1}{2}} P_{2}\left(2^{-\frac{1}{2}}(u+v)\right) a \\
& =2 P_{2}\left(2^{-\frac{1}{2}}(u+v)\right) a=a .
\end{aligned}
$$


Hence, the mappings described in (i) are bijections between $A_{2}(u)$ and $A_{2}\left(2^{-\frac{1}{2}}(u+v)\right)$, and are inverses of each other. Moreover, by Lemma 2.5 (vi),

$$
\left.2^{\frac{1}{2}} P_{2}\left(2^{-\frac{1}{2}}(u+v)\right)\right|_{A_{2}(u)}=\left.2^{-\frac{1}{2}}\left(\operatorname{id}_{A}+\phi_{u, v}\right)\right|_{A_{2}(u)},
$$

which implies that (ii) holds.

It will be shown that the mapping $\left.2^{\frac{1}{2}} P_{2}\left(2^{-\frac{1}{2}}(u+v)\right)\right|_{A_{2}(u)}$ is a Jordan*-isomorphism from the $\mathrm{JBW}^{*}$-algebra $A_{2}(u)$ to the $\mathrm{JBW}^{*}$-algebra $A_{2}\left(2^{-\frac{1}{2}}(u+v)\right)$. To this end, let $a$ be an element of $A_{2}(u)$, and, since $P_{2}\left(2^{-\frac{1}{2}}(u+v)\right)$ is a structural projection, using (5),

$$
\begin{aligned}
\left\{2^{\frac{1}{2}} P_{2}\left(2^{-\frac{1}{2}}(u+v)\right) a\right. & \left.2^{-\frac{1}{2}}(u+v) P_{2}\left(2^{-\frac{1}{2}}(u+v)\right) a\right\} \\
= & 2 P_{2}\left(2^{-\frac{1}{2}}(u+v)\right)\left\{a P_{2}\left(2^{-\frac{1}{2}}(u+v)\right) 2^{-\frac{1}{2}}(u+v) a\right\} \\
= & 2^{\frac{1}{2}} P_{2}\left(2^{-\frac{1}{2}}(u+v)\right)\{a u+v a\} \\
= & 2^{\frac{1}{2}} P_{2}\left(2^{-\frac{1}{2}}(u+v)\right)\{a u a\},
\end{aligned}
$$

since, by (10),

$$
\{a v a\} \in\left\{A_{2}(u) A_{1}(u) A_{2}(u)\right\}=\{0\} .
$$

It follows that the mapping is a Jordan isomorphism. Moreover, since $P_{2}\left(2^{-\frac{1}{2}}(u+v)\right)$ is a structural projection,

$$
\begin{aligned}
\left\{2^{-\frac{1}{2}}(u+v)\right. & \left.2^{\frac{1}{2}} P_{2}\left(2^{-\frac{1}{2}}(u+v)\right) a 2^{-\frac{1}{2}}(u+v)\right\} \\
= & (2)^{\frac{1}{2}} P_{2}\left(2^{-\frac{1}{2}}(u+v)\right)\left\{2^{-\frac{1}{2}}(u+v) P_{2}\left(2^{-\frac{1}{2}}(u+v)\right) a 2^{-\frac{1}{2}}(u+v)\right\} \\
= & 2^{\frac{1}{2}}\left\{P_{2}\left(2^{-\frac{1}{2}}(u+v)\right) 2^{-\frac{1}{2}}(u+v) a P_{2}\left(2^{-\frac{1}{2}}(u+v)\right) 2^{-\frac{1}{2}}(u+v)\right\} \\
= & 2^{\frac{1}{2}}\left\{2^{-\frac{1}{2}}(u+v) a 2^{-\frac{1}{2}}(u+v)\right\} \\
= & 2^{-\frac{1}{2}}\left(\left\{\begin{array}{lll}
u & a & u
\end{array}\right\}+\left\{\begin{array}{lll}
v a & v
\end{array}+2\{u a v\}\right)\right. \\
= & 2^{-\frac{1}{2}}\left(\left\{\begin{array}{lll}
u & a & u
\end{array}+2\left\{\begin{array}{lll}
u & a & v
\end{array}\right),\right.\right.
\end{aligned}
$$

since, by (10),

$$
\{v a v\} \in\left\{A_{2}(v) A_{1}(v) A_{2}(v)\right\}=\{0\} .
$$

Furthermore, using Lemma 2.5 (vi), the fact that $\phi_{u, v}$ is a triple automorphism, Lemma 2.3 (i), and (1),

$$
\begin{aligned}
& 2^{\frac{1}{2}} P_{2}\left(2^{-\frac{1}{2}}(u+v)\right)\{u a u\}=2^{-\frac{1}{2}}\left(\operatorname{id}_{A}+\phi_{u, v}\right)\{u a u\} \\
& =2^{-\frac{1}{2}}\left(\{u a u\}+\left\{v \phi_{u, v} a v\right\}\right) \\
& =2^{-\frac{1}{2}}\left(\left\{\begin{array}{lll}
u & a & u
\end{array}\right\}+2\left\{v\left\{\begin{array}{lll}
v & u & a
\end{array}\right\} v\right\}\right. \\
& =2^{-\frac{1}{2}}(\{u a u\}+2\{u a v\}) \text {. }
\end{aligned}
$$


It follows from $(17),(18)$ that the mapping $2^{\frac{1}{2}} P_{2}\left(2^{-\frac{1}{2}}(u+v)\right)$ is a Jordan *-isomorphism from the $\mathrm{JBW}^{*}$-algebra $A_{2}(u)$ onto the $\mathrm{JBW}^{*}$-algebra $A_{2}\left(2^{-\frac{1}{2}}(u+v)\right)$ and, clearly, its inverse has the same property. Since the Jordan triple product in both of these $\mathrm{JBW}^{*}$-algebras coincide with the restrictions of the triple product on $A$, this completes the proof of (i).

By applying (i) to the tripotent $v$ rather than $u$ it follows that the mapping $\left.2^{\frac{1}{2}} P_{2}(v) 2^{\frac{1}{2}} P_{2}\left(2^{-\frac{1}{2}}(u+v)\right)\right|_{A_{2}(u)}$ is a Jordan *-isomorphism from the JBW*-algebra $A_{2}(u)$ onto the $\mathrm{JBW}^{*}$-algebra $A_{2}(v)$. Observe that, by Lemma 2.5 (vi), the compatibility of $u$ and $v$, and (15),

$$
\begin{aligned}
2 P_{2}(v) P_{2}\left(2^{-\frac{1}{2}}(u+v)\right) P_{2}(u) & =P_{2}(v)\left(\operatorname{id}_{A}+\phi_{u, v}\right) P_{2}(u) \\
& =P_{2}(v) P_{2}(u)+\phi_{u, v} P_{2}(u) P_{2}(u)=\phi_{u, v} P_{2}(u),
\end{aligned}
$$

and the proof of (iii) is complete.

Since triple isomorphisms on $\mathrm{JBW}^{*}$-triples are automatically isometric the result above has the following immediate corollary.

Corollary 2.7. Under the conditions of Theorem 2.6, for all elements a in $A_{2}(u)$,

$$
2\|a\|^{2}=\left\|a+\phi_{u, v} a\right\|^{2} .
$$

\section{Decoherent states}

Let $A$ be a $\mathrm{JBW}^{*}$-triple with predual $A_{*}$, and let $A_{1}$ and $A_{* 1}$ be the closed unit balls in $A$ and $A_{*}$, respectively. For each subset $E$ of $A_{* 1}$ and $F$ of $A_{1}$, let the subsets $E^{\prime}$ and $F$, be defined by

$$
E^{\prime}=\left\{a \in A_{1}: a(x)=1 \forall x \in E\right\}, \quad F_{\prime}=\left\{x \in A_{* 1}: a(x)=1 \forall a \in F\right\} .
$$

Then $E^{\prime}$ is a weak*-closed face of $A_{1}$ and $F_{l}$ is a norm-closed face of $A_{* 1}$ and the mappings $E \mapsto E^{\prime}$ and $F \mapsto F$, are anti-order isomorphisms between the complete lattices $\mathcal{F}_{n}\left(A_{* 1}\right)$ and $F_{w^{*}}\left(A_{1}\right)$ of norm-closed faces of $A_{* 1}$ and weak*-closed faces of $A_{1}$ and are inverses of each other. The mapping $u \mapsto\{u\}$, is an order isomorphism from the complete lattice of tripotents $\tilde{\mathcal{U}}(A)$ in $A$ onto the complete lattice $\mathcal{F}_{n}\left(A_{* 1}\right)$. Moreover, the mapping $u \mapsto\{u\}\}^{\prime}$ is an anti-order-isomorphism from $\tilde{\mathcal{U}}(A)$ onto the complete lattice $\mathcal{F}_{w^{*}}\left(A_{1}\right)$. Furthermore, for every element $u$ in $\mathcal{U}(A)$,

$$
\{u\}_{\prime}^{\prime}=u+A_{0}(u) \cap A_{1} .
$$

The reader is referred to [13] for details.

Lemma 3.1. Let $A$ be a $J B W^{*}$-triple with predual $A_{*}$, let $\phi$ be an element of the group $\operatorname{Aut}(A)$ of triple automorphisms of $A$ with pre-adjoint the element $\phi_{*}$ of the 
group $\operatorname{Aut}\left(A_{*}\right)$ of linear isometries of $A_{*}$, and let $u$ be an element of the partially ordered set $\mathcal{U}(A)$ of tripotents in $A$ with $\{u\}$, the corresponding norm-closed face of the unit ball $A_{* 1}$ in $A_{*}$. Then,

$$
\phi_{*}\left(\{u\}_{\prime}\right)=\left\{\phi^{-1} u\right\}_{\prime} .
$$

Proof. For each element $y$ in $\phi_{*}\left(\{u\}_{1}\right)$, there exists an element $x$ in $\{u\}$, such that

$$
\phi_{*} x=y .
$$

Then,

$$
1=x(u)=\left(\phi_{*}\right)^{-1} y(u)=\left(\phi^{-1}\right)_{*} y(u)=y\left(\phi^{-1} u\right),
$$

and $y$ lies in the norm-closed face $\left\{\phi^{-1} u\right\}_{1}$. Hence, $\phi_{*}\left(\{u\}_{1}\right)$ is contained in $\left\{\phi^{-1} u\right\}_{1}$. The reverse inclusion follows by a simple reversion of the argument above.

The following corollary is immediate from Lemma 3.1.

Corollary 3.2. Let $A$ be a $J B W^{*}$-triple with predual $A_{*}$, let $u$ and $v$ be a pair of rigidly collinear tripotents in $A$, let $\phi_{u, v}$ be the exchange automorphism associated with $u$ and $v$ having pre-adjoint the involutive linear isometry $\left(\phi_{u, v}\right)_{*}$ of $A_{*}$, and let $\{u\}$, and $\{v\}$, be the corresponding norm-closed faces of the unit ball $A_{* 1}$ in $A_{*}$. Then,

$$
\left(\phi_{u, v}\right)_{*}\left(\{u\}_{1}\right)=\{v\}_{\prime} .
$$

For every element $x$ in $A_{*}$ there exists a unique tripotent $e(x)$ in $A$ such that $x$ lies in $A_{2}(e(x))_{*}$ and the restriction of $x$ to $A_{2}(e(x))$ is a faithful normal positive linear functional on the $\mathrm{JBW}^{*}$-algebra $A_{2}(e(x))$. The tripotent $e(x)$ is said to be the support of $x$. The support $e(x)$ of an element $x$ in $A_{*}$ is the least tripotent in $A$ such that $x(e(x))$ equals $\|x\|$. If $x$ is of norm one then the face $\{x\}^{\prime}$, of $A_{1}$ coincides with the norm-closed face $\{e(x)\}$, which is the normal state space of the $\mathrm{JBW}^{*}$-algebra $A_{2}(e(x))$. From a physical point of view, $\{e(x)\}$, may be regarded as the set of normalized states of the local system defined by the normalized state $x$. For details the reader is referred to $[13,23]$.

Lemma 3.3. Let $A$ be a $J B W^{*}$-triple with predual $A_{*}$, let $x$ be an element of $A_{*}$ of norm one, having support tripotent $e(x)$, and let $A_{2}(e(x))_{*}$ be the structural subspace of $A_{*}$ that is the predual of the Peirce space $A_{2}(e(x))$ of A corresponding to $e(x)$. Then, $A_{2}(e(x))_{*}$ is the smallest structural subspace of $A_{*}$ containing $x$.

Proof. Since the norm-closed face $\{x\}^{\prime}$, is the normal state space of the JBW*-algebra $A_{2}(e(x))$, it follows that $x$ lies in $A_{2}(e(x))_{*}$. Suppose that $J$ is a weak ${ }^{*}$-closed inner ideal in $A$ for which $x$ lies in the predual $J_{*}$ of $J$. Then, by [12, Lemma 5.2 and Theorem 5.4], the tripotent $e(x)$ lies in $J$. Hence, $A_{2}(e(x))$ is contained in $J$ and $A_{2}(e(x))_{*}$ is contained in $J_{*}$, as required. 
Two elements $x$ and $y$ of norm one in the pre-symmetric space $A_{*}$ are said to be decoherent if the pair $A_{2}(e(x))_{*}$ and $A_{2}(e(y))_{*}$ of structural subspaces are rigidly collinear. The following result is an immediate consequence of Lemma 3.3 and results of $[10,12]$.

Lemma 3.4. Let $A$ be a $J B W^{*}$-triple, let $x$ and $y$ be elements of the predual $A_{*}$ of $A$ of norm one, let $e(x)$ and $e(y)$ be the support tripotents of $x$ and $y$, and let $A_{2}(e(x))$ and $A_{2}(e(y))$ and $P_{2}(e(x))$ and $P_{2}(e(y))$ be Peirce spaces and Peirce projections associated with $e(x)$ and $e(y)$ respectively. Then, the following are equivalent.

(i) $x$ and $y$ form a decoherent pair.

(ii) The tripotents $e(x)$ and $e(y)$ form a rigidly collinear pair.

(iii) The weak*-closed inner ideals $A_{2}(e(x))$ and $A_{2}(e(y))$ in $A$ form a rigidly collinear pair.

(iv) The structural projections $P_{2}(e(x))_{*}$ and $P_{2}(e(y))_{*}$ on $A_{*}$ form a rigidly collinear pair.

The following result is now immediate from Lemma 2.4 and Theorem 2.6.

Theorem 3.5. Let $A$ be a $J B W^{*}$-triple with predual $A_{*}$, let $x$ and $y$ be a decoherent pair of elements of $A_{*}$ of norm one, having support tripotents $e(x)$ and $e(y)$, let $\phi_{e(x), e(y)}$ be the associated exchange automorphism, and let $A_{2}(e(x))_{*}$ and $A_{2}(e(y))_{*}$ and $P_{2}(e(x))_{*}$ and $P_{2}(e(y))_{*}$ be the corresponding structural subspaces and structural projections, respectively. Then, the following results hold.

(i) $A_{2}(e(x))_{*} \oplus A_{2}(e(y))_{*}$ is the smallest structural subspace of $A_{*}$ containing $x$ and $y$.

(ii) $A_{2}(e(x+y))_{*} \subseteq A_{2}(e(x))_{*} \oplus A_{2}(e(y))_{*}$.

(iii) The structural subspaces $A_{2}(e(x))_{*}, A_{2}(e(y))_{*}$ and $A_{2}(e(x))_{*} \oplus A_{2}(e(y))_{*}$ form a pairwise compatible family.

(iv) The linear isometry $\left(\phi_{e(x), e(y)}\right)_{*}$ maps the structural subspace $A_{2}(e(x))_{*}$ onto the structural subspace $A_{2}(e(y))_{*}$.

(v) The mappings

$$
\left.2^{\frac{1}{2}} P_{2}\left(2^{-\frac{1}{2}}(e(x)+e(y))\right)_{*}\right|_{A_{2}(e(x))_{*}}: A_{2}(e(x))_{*} \longrightarrow A_{2}\left(2^{-\frac{1}{2}}(e(x)+e(y))\right)_{*}
$$

and

$$
\left.2^{\frac{1}{2}} P_{2}(e(x))_{*}\right|_{A_{2}\left(2^{-\frac{1}{2}}(e(x)+e(y))\right)_{*}}: A_{2}\left(2^{-\frac{1}{2}}(e(x)+e(y))\right)_{*} \longrightarrow A_{2}(e(x))_{*}
$$

are isometric linear isomorphisms onto the structural subspaces $A_{2}\left(2^{-\frac{1}{2}}(e(x)+\right.$ $e(y)))_{*}$ and $A_{2}(e(x))_{*}$, respectively, and are inverses of each other. 
(vi) The equality

$$
\left.2^{\frac{1}{2}} P_{2}\left(2^{-\frac{1}{2}}(e(x)+e(y))\right)_{*}\right|_{A_{2}(e(x))_{*}}=\left.2^{-\frac{1}{2}}\left(\operatorname{id}_{A}+\phi_{e(x), e(y)}\right)_{*}\right|_{A_{2}(e(x))_{*}}
$$

holds.

(vii) The equalities

$$
\begin{aligned}
\left.\left(\phi_{e(x), e(y)}\right)_{*}\right|_{A_{2}(e(x))_{*}} & =\left.2 D(e(y), e(x))_{*}\right|_{A_{2}(e(x))_{*}} \\
& =\left.2 P_{2}(e(y))_{*} P_{2}\left(2^{-\frac{1}{2}}(e(x)+e(y))\right)_{*}\right|_{A_{2}(e(x))_{*}}
\end{aligned}
$$

hold.

Before coming to the main result the following lemma is needed.

Lemma 3.6. Let $A$ be a $J B W^{*}$-triple, let $u$ and $v$ be a pair of collinear tripotents in $A$, let $\{u\}$, and $\{v\}$, be the corresponding norm-closed faces of the unit ball $A_{* 1}$ in the pre-dual $A_{*}$ of $A$, and let $x$ and $y$ be elements of $\{u\}_{1}$, and $\{v\}_{1}$, respectively. Then, the following results hold.

(i) $\|x+y\| \geq 2^{\frac{1}{2}}$.

(ii) Equality holds in the inequality above if and only the support tripotent $e(x+y)$ of $x+y$ is majorized by the tripotent $2^{-\frac{1}{2}}(u+v)$.

Proof. (i) Observe that, being a non-zero tripotent, $2^{-\frac{1}{2}}(u+v)$ is of norm one. However,

$$
\begin{aligned}
(x+y)\left(2^{-\frac{1}{2}}(u+v)\right) & =2^{-\frac{1}{2}}(x(u)+x(v)+y(u)+y(v)) \\
& =2^{-\frac{1}{2}}\left(1+P_{2}(u)_{*} x\left(P_{1}(u) v\right)+P_{2}(v)_{*}\left(P_{1}(v) u\right)+1\right)=2^{\frac{1}{2}} .
\end{aligned}
$$

Therefore,

$$
\|x+y\| \geq 2^{\frac{1}{2}}
$$

as required.

(ii) Suppose that equality holds and then, as above,

$$
(x+y)\left(2^{-\frac{1}{2}}(u+v)\right)=\|x+y\|,
$$

and

$$
e(x+y) \leq 2^{-\frac{1}{2}}(u+v) .
$$

Conversely, if this holds then

$$
(x+y) /\|x+y\| \in\{e(x+y))\}, \subseteq\left\{2^{-\frac{1}{2}}(u+v)\right\},
$$

and

$$
\|x+y\|=2^{-\frac{1}{2}}(x+y)(u+v)=2^{\frac{1}{2}},
$$

as required. 
A decoherent pair $x$ and $y$ of elements of the pre-symmetric space $A_{*}$ of norm one is said to be strongly decoherent when the exchange automorphism $\left(\phi_{e(x), e(y)}\right)_{*}$ of $A_{*}$ maps $x$ to $y$. The main result of the section discussed the conditions under which this occurs. The following lemma considers a more general situation in which $u$ and $v$ form a rigidly collinear pair of tripotents in the $\mathrm{JBW}^{*}$-triple $A$ and $x$ and $y$ are elements of the norm-closed faces $\{u\}$, and $\{v\}$, respectively.

Lemma 3.7. Let $A$ be a $J B W^{*}$-triple, let $u$ and $v$ be a pair of rigidly collinear tripotents in $A$, let $\phi_{u, v}$ be the corresponding exchange automorphism of $A$, let $\{u\}$, and $\{v\}$, be the corresponding norm-closed faces of the unit ball $A_{* 1}$ in the pre-dual $A_{*}$ of $A$, and let $x$ and $y$ be elements of $\{u\}$, and $\{v\}$, respectively. Then, the following conditions are equivalent.

(i) $\|x+y\|=2^{\frac{1}{2}}$.

(ii) $\left(\phi_{u, v}\right)_{*} x=y$.

(iii) $e(x+y) \leq 2^{-\frac{1}{2}}(u+v)$.

(iv) $2 D(u, v)_{*} x=y$.

(v) $2 D(v, u)_{*} y=x$.

Proof. (i) $\Rightarrow\left(\right.$ ii) Observe that, since $y$ lies in $A_{2}(v)_{*}$ and $u$ lies in $A_{1}(v)$,

$$
y(u)=P_{2}(v)_{*} y\left(P_{1}(v) u\right)=0,
$$

and, similarly,

$$
x(v)=0 .
$$

Therefore,

$$
\begin{aligned}
2^{\frac{1}{2}} & =\|x+y\| \geq\left\|P_{2}\left(2^{-\frac{1}{2}}(u+v)\right)_{*}(x+y)\right\| \\
& =\sup \left\{\mid(x+y)\left(P_{2}\left(2^{-\frac{1}{2}}(u+v)\right) a \mid: a \in A_{1}\right\}\right. \\
& \geq \sup \left\{|(x+y) a|: a \in A_{2}\left(2^{-\frac{1}{2}}(u+v)\right)_{1}\right\} \geq\left|(x+y)\left(2^{-\frac{1}{2}}(u+v)\right)\right| \\
& =\left|2^{-\frac{1}{2}}(x(u)+x(v)+y(u)+y(v))\right|=2^{\frac{1}{2}} .
\end{aligned}
$$

Hence,

$$
\left\|P_{2}\left(2^{-\frac{1}{2}}(u+v)\right)_{*}(x+y)\right\|=\|x+y\|,
$$

and, since $P_{2}\left(2^{-\frac{1}{2}}(u+v)\right)_{*}$ is a structural projection,

$$
P_{2}\left(2^{-\frac{1}{2}}(u+v)\right)_{*}(x+y)=x+y .
$$

It follows from (16) that

$$
\left(\phi_{u, v}\right)_{*}(x+y)=x+y .
$$


and, using Corollary 3.2 and Lemma 2.2,

$$
x-\left(\phi_{u, v}\right)_{*} y=\left(\phi_{u, v}\right)_{*} x-y \in A_{2}(u)_{*} \cap A_{2}(v)_{*}=\{0\} .
$$

Hence,

$$
\left(\phi_{u, v}\right)_{*} x=y,
$$

as required.

(ii) $\Rightarrow$ (i) Observe that

$$
\left(\phi_{u, v}\right)_{*}(x+y)=x+\left(\phi_{u, v}\right)_{*} x=x+y .
$$

Therefore, using Lemma 2.3 (i) and (16),

$$
x+y \in\left(A_{2}(u)_{*} \oplus A_{2}(v)_{*}\right) \cap\left(A_{2}\left(2^{-\frac{1}{2}}(u+v)\right)_{*} \oplus A_{0}\left(2^{-\frac{1}{2}}(u+v)\right)_{*}\right) .
$$

Therefore, there exist elements $z_{2}$ in $A_{2}\left(2^{-\frac{1}{2}}(u+v)\right)_{*}$ and $z_{0}$ in $A_{0}\left(2^{-\frac{1}{2}}(u+v)\right)_{*}$ such that

$$
x+y=z_{2}+z_{0} .
$$

By Lemma $2.5(\mathrm{i}), A_{2}\left(2^{-\frac{1}{2}}(u+v)\right)_{*}$ is contained in $A_{2}(u)_{*} \oplus A_{2}(v)_{*}$, and, therefore,

$$
z_{0}=x+y-z_{2} \in\left(A_{2}(u)_{*} \oplus A_{2}(v)_{*}\right) \cap A_{0}\left(2^{-\frac{1}{2}}(u+v)\right)_{*} .
$$

It follows from [12, Lemma 5.2 and Theorem 5.4], that the support tripotent $e\left(z_{0}\right)$ of $z_{0}$ is contained in the weak ${ }^{*}$-closed inner ideal $\left(A_{2}(u) \oplus A_{2}(v)\right) \cap A_{0}\left(2^{-\frac{1}{2}}(u+v)\right)$. Therefore, by Lemma 2.5 (ii), $e\left(z_{0}\right)$ is equal to zero which implies that $z_{0}$ is equal to zero. It follows that the element $x+y$ lies in the predual $A_{2}\left(2^{-\frac{1}{2}}(u+v)\right)_{*}$ of the JBW*-algebra $A_{2}\left(2^{-\frac{1}{2}}(u+v)\right)$.

Let $a$ be an element of the JBW*-algebra $A_{2}\left(2^{-\frac{1}{2}}(u+v)\right)$. Then, by Theorem 2.6 (i), there exists an element $b$ of $A_{2}(u)$ such that

$$
a=2^{\frac{1}{2}} P_{2}\left(2^{-\frac{1}{2}}(u+v)\right) b .
$$

Since, by Lemma 2.3 (i),

$$
\phi_{u, v} b \in A_{2}(v) \subseteq A_{1}(u),
$$

using Lemma 2.5 (vi), (14), and Lemma 2.3 (i), it can be seen that

$$
\begin{aligned}
(x+y)(a) & =2^{\frac{1}{2}}\left(x+\left(\phi_{u, v}\right)_{*} x\right)\left(P_{2}\left(2^{-\frac{1}{2}}(u+v)\right) b\right) \\
& \left.=2^{-\frac{1}{2}}\left(\operatorname{id}_{A_{*}}+\left(\phi_{u, v}\right)_{*}\right) x\left(\operatorname{id}_{A}+\phi_{u, v}\right) b\right) \\
& =2^{\frac{1}{2}}\left(x(b)+x\left(\phi_{u, v} b\right)\right) \\
& =2^{\frac{1}{2}} x(b)+2^{\frac{1}{2}}\left(P_{2}(u)_{*} x\right)\left(P_{1}(u) \phi_{u, v} b\right)=2^{\frac{1}{2}} x(b) .
\end{aligned}
$$


As in the proof of Theorem 2.6, $2^{\frac{1}{2}} P_{2}\left(2^{-\frac{1}{2}}(u+v)\right)$ is a Jordan *-isomorphism between $\mathrm{JBW}^{*}$-algebras and, therefore, maps positive elements of $A_{2}(u)$ to positive elements of $A_{2}\left(2^{-\frac{1}{2}}(u+v)\right)$. Since $x$ is a state of the JBW*-algebra $A_{2}(u)$, it follows from (19) that $x+y$ is a positive linear functional on the $\mathrm{JBW}^{*}$-algebra $A_{2}\left(2^{-\frac{1}{2}}(u+v)\right)$. Therefore, observing that, as in the proof above,

$$
x(v)=y(u)=0,
$$

and, using the fact that a positive linear functional on a $\mathrm{JBW}^{*}$-algebra attains its norm at the unit,

$$
\|x+y\|=(x+y)\left(2^{-\frac{1}{2}}(u+v)\right)=\left(2^{-\frac{1}{2}}\right)(x(u)+y(v))=2^{\frac{1}{2}},
$$

as required.

(ii) $\Leftrightarrow$ (iii) $\quad$ This is immediate from Lemma 3.6.

(ii) $\Rightarrow$ (iv) Observe that, since $u$ and $v$ are compatible, using (7)-(10), for an element $a$ in $A$,

$$
\begin{aligned}
& D(u, v) P_{2}(u) a=\left\{u v P_{2}(u) a\right\} \in\left\{A_{2}(u) A_{1}(u) A_{2}(u)\right\}=\{0\}, \\
& D(u, v) P_{2}(v) a=\left\{u v P_{2}(v) a\right\} \in A_{2}(u) \cap A_{1}(v)=A_{2}(u), \\
& D(u, v) P_{1}(u) P_{1}(v) a=\left\{u v P_{1}(u) P_{1}(v) a\right\} \in A_{2}(u) \cap A_{0}(v)=\{0\}, \\
& D(u, v) P_{1}(u) P_{0}(v) a=\left\{u v P_{1}(u) P_{0}(v) a\right\}=0, \\
& D(u, v) P_{0}(u) P_{1}(v) a=\left\{u v P_{0}(u) P_{1}(v) a\right\} \in A_{1}(u) \cap A_{0}(v), \\
& D(u, v) P_{0}(u) P_{0}(v) a=\left\{u v P_{0}(u) P_{0}(v) a\right\}=0 .
\end{aligned}
$$

Therefore, since $x$ lies in $A_{2}(u)_{*}$, using Lemma 2.4, (20)-(25), (15), and Lemma 2.3 (i),

$$
\begin{aligned}
2 D(u, v)_{*} x(a)= & 2 x(D(u, v) a)=2 P_{2}(u)_{*} x(D(u, v) a) \\
= & 2 P_{2}(u)_{*} x\left(D ( u , v ) \left(P_{2}(u)+P_{2}(v)+P_{1}(u) P_{1}(v)\right.\right. \\
& \left.\left.+P_{1}(u) P_{0}(v)+P_{0}(u) P_{1}(v)+P_{0}(u) P_{0}(v)\right) a\right) \\
= & 2 x\left(P_{2}(u)\left(D(u, v) P_{2}(v) a+D(u, v) P_{0}(u) P_{1}(v)\right) a\right) \\
= & 2 x\left(D(u, v) P_{2}(v) a+D(u, v) P_{2}(v) P_{0}(u) P_{1}(v) a\right)=x\left(\phi_{u, v} P_{2}(v) a\right) \\
= & \left(\phi_{u, v}\right)_{*} x\left(P_{2}(v) a\right)=y\left(P_{2}(v) a\right)=P_{2}(v)_{*} y(a) \\
= & y(a),
\end{aligned}
$$

as required.

(iv) $\Rightarrow$ (ii) For each element $a$ in $A$, by (15) and Lemma 2.3 (i),

$$
\begin{aligned}
\left(\phi_{u, v}\right)_{*} x(a) & =x\left(\phi_{u, v} a\right)=\left(P_{2}(u)_{*} x\right)\left(\phi_{u, v} a\right) \\
& =x\left(P_{2}(u) \phi_{u, v} a\right)=x\left(\phi_{u, v} P_{2}(v) a\right) \\
& =2 x\left(D(u, v) P_{2}(v) a\right)=2 D(u, v)_{*} x\left(P_{2}(v) a\right) \\
& =y\left(P_{2}(v) a\right)=P_{2}(v)_{*} y(a)=y(a),
\end{aligned}
$$


as required.

(ii) $\Leftrightarrow(\mathrm{v}) \quad$ This follows by exchanging $u$ and $v$ in the argument above.

It is now possible to state the main result of the paper.

Theorem 3.8. Let $A$ be a $J B W^{*}$-triple with predual $A_{*}$, let $x$ and $y$ be a decoherent pair of elements of $A_{*}$ of norm one, let $e(x), e(y)$, and $e(x+y)$ be the support tripotents of $x, y$, and $x+y$, respectively, and let $\phi_{e(x), e(y)}$ be the associated exchange automorphism of $A$. Then, the following conditions are equivalent.

(i) The elements $x$ and $y$ are strongly decoherent.

(ii) $\|x+y\|=2^{\frac{1}{2}}$.

(iii) $e(x+y) \leq 2^{-\frac{1}{2}}(e(x)+e(y))$.

(iv) $e(x+y)=2^{-\frac{1}{2}}(e(x)+e(y))$.

(v) $2 D(e(x), e(y))_{*} x=y$.

(vi) $2 D(e(y), e(x))_{*} y=x$.

Proof. All except the implication (iii) $\Rightarrow$ (iv) follows from Lemma 3.7. Let

$$
f=2^{-\frac{1}{2}}(e(x)+e(y))-e(x+y) .
$$

Then, $f$ is a tripotent majorized by the tripotent $2^{-\frac{1}{2}}(e(x)+e(y))$, and, therefore, is a self-adjoint idempotent in the $\mathrm{JBW}^{*}$-algebra $A_{2}\left(2^{-\frac{1}{2}}(e(x)+e(y))\right)$. Therefore, by Theorem 2.6, $2^{\frac{1}{2}} P_{2}(e(x)) f$ and $2^{\frac{1}{2}} P_{2}(e(y)) f$ are self-adjoint idempotents in the $\mathrm{JBW}^{*}$-algebras $A_{2}(e(x))$ and $A_{2}(e(y))$, respectively. Hence, again using the fact that

$$
x(e(y))=y(e(x))=0,
$$

it follows that

$$
\begin{aligned}
x\left(2^{\frac{1}{2}} P_{2}(e(x)) f\right)+y\left(2^{\frac{1}{2}} P_{2}(e(y)) f\right)= & 2^{\frac{1}{2}}\left(P_{2}(e(x))_{*} x\right)(f)+P_{2}(e(y))_{*} y(f) \\
= & 2^{\frac{1}{2}}(x+y)(f) \\
= & (x+y)(e(x)+e(y)) \\
& -2^{\frac{1}{2}}(x+y)(e(x+y)) \\
= & x(e(x))+y(e(y))-2^{\frac{1}{2}}\|x+y\| \\
= & 0 .
\end{aligned}
$$

Since $x$ and $y$ are faithful states of the $\mathrm{JBW}^{*}$-algebras $A_{2}(e(x))$ and $A_{2}(e(y))$, respectively, by (26),

$$
x\left(2^{\frac{1}{2}} P_{2}(e(x)) f\right)=y\left(2^{\frac{1}{2}} P_{2}(e(y)) f\right)=0,
$$


and, hence,

$$
P_{2}(e(x)) f=P_{2}(e(y)) f=0 .
$$

It follows from Theorem 2.6 that,

$$
f=2 P_{2}\left(2^{-\frac{1}{2}}(e(x)+e(y)) P_{2}(e(x)) f=0,\right.
$$

and

$$
e(x+y)=2^{-\frac{1}{2}}(e(x)+e(y))
$$

as required.

Before examining some consequences of this result, two further preliminary results are needed.

Lemma 3.9. Let $A$ be a $J B W^{*}$-triple with predual $A_{*}$, let $x$ be an element of $A_{*}$ of norm one having support tripotent $e(x)$, and let $\phi$ be an element of the group $\operatorname{Aut}(A)$ of triple automorphisms of $A$ having pre-adjoint the linear isometry $\phi_{*}$ of $A_{*}$. Then,

$$
e\left(\phi_{*} x\right)=\phi^{-1}(e(x)) \text {. }
$$

Proof. Notice that $\phi^{-1}(e(x))$ is a tripotent and the element $\phi_{*} x$ of $A_{*}$ is of norm one. Moreover,

$$
\phi_{*} x\left(\phi^{-1}(e(x))=x(e(x))=1,\right.
$$

and it follows that the tripotent $\phi^{-1}(e(x))$ is contained in the norm-closed face $\left\{\phi_{*} x\right\}^{\prime}$ of the unit ball $A_{* 1}$ in $A_{*}$. Therefore,

$$
e\left(\phi_{*} x\right) \leq \phi^{-1}(e(x)) \text {. }
$$

Applying the same result to the element $\phi_{*} x$ and the triple automorphism $\phi^{-1}$ shows that

$$
e(x)=e\left(\left(\phi_{*}\right)^{-1} \phi_{*} x\right)=e\left(\left(\phi^{-1}\right)_{*} \phi_{*} x\right) \leq \phi\left(e\left(\phi_{*} x\right)\right) .
$$

Since the restrictions of triple automorphisms of $A$ are order isomorphisms of the partially ordered set $\mathcal{U}(A)$,

$$
\phi^{-1}(e(x)) \leq \phi^{-1} \phi\left(e\left(\phi_{*} x\right)\right)=e\left(\phi_{*} x\right),
$$

and the result follows from (27), (28).

Lemma 3.10. Let $A$ be a $J B W^{*}$-triple, let $u$ and $v$ be a rigidly collinear pair of tripotents in $A$, let $\phi_{u, v}$ be the associated exchange automorphism of $A$, and let $f$ be a tripotent in the Peirce space $A_{2}(u)$ corresponding to $u$. Then, $f$ and $\phi_{u, v} f$ form $a$ rigidly collinear pair of tripotents in $A$. 
Proof. Observe that, by Lemma 2.3 (i), (2), and (10),

$$
\begin{aligned}
\left\{f f \phi_{u, v} f\right\} & =2\{f f D(v, u) f\}=2 D(f, f) D(v, u) f \\
& =2 D(v, u) D(f, f) f+2 D(f,\{f v u\}) f-2 D(\{v u f\}, f) f \\
& =2 D(v, u) f-\left\{f f \phi_{u, v} f\right\},
\end{aligned}
$$

since

$$
\{f v u\} \in\left\{A_{2}(u) A_{1}(u) A_{2}(u)\right\}=\{0\} .
$$

Again using Lemma 2.3 (i), it follows that

$$
\left\{f f \phi_{u, v} f\right\}=D(v, u) f=\frac{1}{2} \phi_{u, v} f,
$$

which implies that the tripotent $\phi_{u, v} f$ lies in $A_{1}(e)$. Furthermore, since $\phi_{u, v}$ is a triple automorphism of period two,

$$
\left\{\phi_{u, v} f \phi_{u, v} f f\right\}=\phi_{u, v}\left\{f f \phi_{u, v} f\right\}=\frac{1}{2} \phi_{u, v} \phi_{u, v} f=\frac{1}{2} f,
$$

and the tripotent $f$ lies in $A_{1}\left(\phi_{u, v} f\right)$. Hence, $f$ and $\phi_{u, v}$ form a collinear pair with $f$ in $A_{2}(u)$ and $\phi_{u, v} f$ in $\phi_{u, v} A_{2}(u)$, which, by Lemma 2.3 (i), coincides with $A_{2}(v)$. Therefore, using Lemma 2.2,

$$
A_{2}(f) \cap A_{2}\left(\phi_{u, v} f\right) \subseteq A_{2}(u) \cap A_{2}(v)=\{0\},
$$

and, by the same lemma, $f$ and $\phi_{u, v} f$ form a rigidly collinear pair.

A further consequence of Lemmas 3.7, 3.9, and 3.10 can now be stated.

Theorem 3.11. Let $A$ be a $J B W^{*}$-triple, let $u$ and $v$ be a pair of rigidly collinear tripotents in $A$ and let $\{u\}$, and $\{v\}$, be the corresponding norm-closed faces of the unit ball $A_{* 1}$ in the pre-dual $A_{*}$ of $A$. Then, for each element $x$ of $\{u\}$, there exists a unique element $y$ in $\{v\}$, such that $x$ and $y$ form a strongly decoherent pair.

Proof. Observe that, since $x$ is contained in $\{u\}_{\text {, }}$

$$
e(x) \leq u \text {. }
$$

Let $\phi_{u, v}$ be the exchange automorphism of $A$ associated with $u$ and $v$, and let $y$ be the element $\left(\phi_{u, v}\right)_{*} x$ of $A_{*}$. Since $\left(\phi_{u, v}\right)_{*}$ is an isometry, $y$ is of norm one, using Lemma 3.9, the fact that the restriction of $\phi_{u, v}$ to $\mathcal{U}(A)$ is an order isomorphism, and (29),

$$
e(y)=e\left(\left(\phi_{u, v}\right)_{*} x\right)=\phi_{u, v} e(x) \leq \phi_{u, v} u=v .
$$

By Lemma 3.10, it follows that $e(x)$ and $e(y)$ form a rigidly collinear pair of tripotents, which, by Lemma 3.7, are strongly decoherent. 
Observe that, by Theorem 3.8,

$$
\left(\phi_{e(x), e(y)}\right)_{*} x=y
$$

Suppose that $z$ is a further element of $\{v\}$, for which $x$ and $z$ form a strongly decoherent pair. Then, by Lemma 3.7,

$$
z=\left(\phi_{u, v}\right)_{*} x=y
$$

and the element $y$ is unique.

\section{Applications and examples}

As was remarked in the introduction, contractive projections play an important part not only in the theory of pre-symmetric spaces but also in their physical applications $[5,18,29,35,40]$. Recall that a $G L$-projection $R$ on the pre-symmetric space $A_{*}$ is a contractive projection such that, if $x$ is an element of $A_{*}$ for which, for all elements $y$ in $A_{*}$

$$
\|x+R y\|=\|x\|+\|R y\|,
$$

then $x$ lies in the kernel of $R$. Observe that such a projection is an element of the complete lattice $\mathcal{S}_{*}\left(A_{*}\right)$ of structural projections on $A_{*}$ provided that, for all elements $x$ in $A_{*}$ for which $\|R x\|$ and $\|x\|$ coincide, then so also do $R x$ and $x$. For every contractive projection $R$ on $A_{*}$ there exists a unique GL-projection with the same range as that of $R$. In particular, for each element $x$ in $A_{*}$ of norm one, the unique GL-projection $Q_{x}$ onto the closed subspace $\mathbb{C} x$ of $A_{*}$ is defined, for $z$ in $A_{*}$, by

$$
Q_{x} z=z(e(x)) x
$$

where $e(x)$ is the support tripotent of $x$. For more details the reader is referred to $[10,26]$.

A family $\left\{x_{j}: j \in \Lambda\right\}$ of elements of norm one in the pre-symmetric space $A_{*}$ is said to be decoherent if each pair of elements in the set is decoherent, and is said to be strongly decoherent if each pair of elements of the set is strongly decoherent. The following result is a consequence of the results of the previous section and makes an important connection between the concepts of strong decoherence and contractive projections.

Theorem 4.1. Let $A$ be a JBW*-triple with predual $A_{*}$ and let $\left\{x_{j}: j \in \Lambda\right\}$ be a decoherent family of elements of $A_{*}$ of norm one. Then, the family is strongly decoherent if and only if there exists a contractive projection on $A_{*}$ with range the norm-closed linear span ${\overline{\operatorname{lin}\left\{x_{j}: j \in \Lambda\right\}}}^{n}$ of the family $\left\{x_{j}: j \in \Lambda\right\}$. 
Proof. If $\Lambda$ contains a single element the result is trivial. Suppose that $\Lambda$ contains two elements $j$ and $k$. Since $x_{j}$ lies in $A_{1}\left(e\left(x_{k}\right)\right)_{*}$ and $x_{k}$ lies in $A_{1}\left(e\left(x_{j}\right)\right)_{*}$, it follows that

$$
2 D\left(e\left(x_{j}\right), e\left(x_{j}\right)\right)_{*} x_{k}=x_{k}, \quad 2 D\left(e\left(x_{k}\right), e\left(x_{k}\right)\right)_{*} x_{j}=x_{j} .
$$

By Theorem 3.8, $x_{j}$ and $x_{k}$ form a strongly decoherent pair if and only if

$$
2 D\left(e\left(x_{j}\right), e\left(x_{k}\right)\right)_{*} x_{j}=x_{k}, \quad 2 D\left(e\left(x_{k}\right), e\left(x_{j}\right)\right)_{*} x_{k}=x_{j} .
$$

The result is now a consequence of [27, Theorem 5.2]. If $\Lambda$ contains more than two elements, then, for an arbitrary pair $j$ and $k$ of elements of $\Lambda$, (30) automatically holds. Moreover, for distinct elements $j, k$, and $l$ in $\Lambda$ and an element $a$ in $A$, since $e\left(x_{j}\right)$ and $e\left(x_{k}\right)$ lie in $A_{1}\left(e\left(x_{l}\right)\right)$, using (8)-(10),

$$
\begin{aligned}
D\left(e\left(x_{j}\right), e\left(x_{k}\right)\right)_{*} x_{l}(a)= & x_{l}\left(P _ { 2 } ( e ( x _ { l } ) ) \left\{e ( x _ { j } ) e ( x _ { k } ) \left(P_{2}\left(e\left(x_{l}\right)\right) a\right.\right.\right. \\
& \left.\left.+P_{1}\left(e\left(x_{l}\right)\right) a+P_{0}\left(e\left(x_{l}\right)\right) a\right)\right\} \\
= & x_{l}\left(P _ { 2 } \left(e\left(x_{l}\right)\left\{e\left(x_{j}\right) e\left(x_{k}\right) P_{2}\left(e\left(x_{l}\right)\right) a\right\}\right.\right. \\
= & 0,
\end{aligned}
$$

since

$$
\begin{aligned}
\left\{e\left(x_{j}\right) e\left(x_{k}\right) P_{2}\left(e\left(x_{l}\right)\right) a\right\} & \in\left\{A_{2}\left(e\left(x_{j}\right)\right) A_{1}\left(e\left(x_{j}\right)\right) A_{1}\left(e\left(x_{j}\right)\right)\right\} \\
& \subseteq A_{2}\left(e\left(x_{j}\right)\right) \subseteq A_{1}\left(e\left(x_{l}\right)\right) .
\end{aligned}
$$

It follows that

$$
D\left(e\left(x_{j}\right), e\left(x_{k}\right)\right)_{*} x_{l}=0 .
$$

Furthermore, by rigid collinearity, (8)-(10), and Lemma 2.2,

$$
\left\{e\left(x_{j}\right) e\left(x_{k}\right) e\left(x_{l}\right)\right\} \in A_{2}\left(e\left(x_{j}\right)\right) \cap A_{2}\left(e\left(x_{l}\right)\right)=\{0\} .
$$

Since (31)-(34) automatically hold, it follows from Theorem 3.8 and [27, Theorem 5.3], that the family is strongly decoherent if and only if its closed linear span is the range of a contractive projection.

This result has a series of corollaries.

Corollary 4.2. Let $u$ and $v$ be a pair of rigidly collinear tripotents in the $J B W^{*}$ triple $A$. Then, there exists a weak*-continuous contractive projection on $A$ with range $\mathbb{C} u \oplus \mathbb{C} v$.

Proof. Let $x$ be an element of the norm-closed face $\{u\}$, of the unit ball $A_{* 1}$ in the predual $A_{*}$ of $A$, let $\phi_{u, v}$ be the exchange automorphism corresponding to $u$ and $v$, and let

$$
y=\left(\phi_{u, v}\right)_{*} x .
$$


Then, by Theorem 3.11, $x$ and $y$ form a strongly decoherent pair. Let $Q_{x}$ and $Q_{y}$ be the unique GL-projections on $A_{*}$ onto $\mathbb{C} x$ and $\mathbb{C} y$ defined in (30). Therefore, using [26] and Theorem 4.1, $Q_{x}+Q_{y}$ is a contractive projection on $A_{*}$ with range $\mathbb{C} x \oplus \mathbb{C} y$. Furthermore, the range of the adjoint $\left(Q_{x}+Q_{y}\right)^{*}$ is clearly $\mathbb{C} e(x)+\mathbb{C} e(y)$. Define the bounded linear projection $R$ on $A_{*}$, for $z$ in $A_{*}$, by

$$
R z=z(u) x+z(v) y .
$$

Then, for an element $a$ in $A$,

$$
R^{*} a=x(a) u+y(a) v .
$$

Moreover, for elements $z$ in $A_{*}$ and $a$ in $A$,

$$
\begin{aligned}
z\left(R^{*}\left(Q_{x}+Q_{y}\right)^{*} a\right) & =\left(\left(Q_{x}+Q_{y}\right)(z(u) x+z(v) y)\right)(a) \\
& =(z(u) x+z(v) y(e(x)) x+z(u) x(e(y)) y+z(v) y)(a) \\
& =(z(u) x+z(u) y)(a)=z\left(R^{*} a\right),
\end{aligned}
$$

and it follows that

$$
R^{*}\left(Q_{x}+Q_{y}\right)^{*}=R^{*} .
$$

Since, by Lemma 3.10, both $e(x)$ and $e(y)$ and $u$ and $v$ form rigidly collinear pairs, the restriction of the norm on $A$ to the two subspaces $\mathbb{C} e(x) \oplus \mathbb{C} e(y)$ and $\mathbb{C} u \oplus \mathbb{C} v$ is Hilbertian. From above, the restriction of $R^{*}$ to $\mathbb{C} e(x) \oplus \mathbb{C} e(y)$ is an isometry between the two Hilbert spaces, and, since $\left(Q_{x}+Q_{y}\right)^{*}$ is contractive, it follows that $R^{*}$ is contractive, as required.

Corollary 4.3. Let $A_{*}$ be a pre-symmetric space and let $\left\{x_{j}: j \in \Lambda\right\}$ be a decoherent family of extreme points of the unit ball $A_{* 1}$ in $A_{*}$. Then, the family $\left\{x_{j}: j \in \Lambda\right\}$ is strongly decoherent.

Proof. Since an extreme point $x$ of $A_{* 1}$ is a minimal norm-closed face, it follows that $e(x)$ is a minimal element of $\mathcal{U}(A)$ and $A_{2}(e(x))$ coincides with $\mathbb{C} e(x)$. Hence, every decoherent pair of extreme points is automatically strongly decoherent.

Observe that, for a rigidly collinear pair $u$ and $v$ of non-minimal tripotents of the $\mathrm{JBW}^{*}$-triple $A$, there exist distinct elements $x$ and $y$ in the norm-closed face $\{u\}$, of the unit ball $A_{* 1}$ in $A_{*}$ for which, by Lemma 3.1, it is necessarily true that $\left(\phi_{u, v}\right)_{*} x$ and $\left.\left(\phi_{u, v}\right)\right)_{*} y$ are distinct elements of $\{v\}_{1_{u}}$. By Theorem 3.11, $x$ and $\left.\left(\phi_{u, v}\right)\right)_{*} x$ form a strongly decoherent pair but $y$ and $\left(\phi_{u, v}\right)_{*} x$ do not. Consequently, by Theorem 4.1 , there is a contractive projection onto the space $\mathbb{C} x \oplus \mathbb{C}\left(\phi_{u, v}\right)_{*} x$ but not onto the space $\mathbb{C} y \oplus \mathbb{C}\left(\phi_{u, v}\right)_{*} x$. An example which illustrates this when $A$ is the $\mathrm{W}^{*}$-algebra $M_{4}(\mathbb{C})$ of $4 \times 4$ complex matrices can be found in [27]. It should also be noted that no such example can be given when $A$ is the $\mathrm{W}^{*}$-algebra $M_{2}(\mathbb{C})$ since rigidly collinear tripotents are of rank one and, therefore, minimal, in which case Corollary 4.3 applies. 
Recall that a $\mathrm{JBW}^{*}$-triple is said to be atomic if, for each non-zero element $u$ of $\mathcal{U}(A)$, there exists a minimal element $e(x)$ of $\mathcal{U}(A)$ for which $e(x) \leq u$. The final corollary shows that, for atomic $\mathrm{JBW}^{*}$-triples, the situation is transparent.

Corollary 4.4. Let $A$ be an atomic $J B W^{*}$-triple with predual $A_{*}$, and let $\left\{u_{j}: j \in \Lambda\right\}$ be a family of pairwise rigidly collinear tripotents in $A$. Then, there exists a weak ${ }^{*}$-continuous contractive projection with range the weak ${ }^{*}$-closed linear span $\overline{\operatorname{lin}\left\{u_{j}: j \in \Lambda\right\}} w^{*}$ of $\left\{u_{j}: j \in \Lambda\right\}$

Proof. Let $k$ lie in $\Lambda$ and let $x_{k}$ be the extreme point of the unit ball $A_{* 1}$ in the predual $A_{*}$ of $A$ such that $e\left(x_{k}\right) \leq u_{k}$. Then, for $j$ not equal to $k$, let

$$
x_{j}=\left(\phi_{u_{k}, u_{j}}\right)_{*} x_{k} .
$$

Since linear isometries clearly map the set of extreme points of $A_{* 1}$ onto itself, by Theorem 3.11, $\left\{x_{j}: j \in \Lambda\right\}$ forms a a decoherent family of extreme points of $A_{*, 1}$. It follows from Corollary 4.3 that the family is strongly decoherent and, by Theorem 4.1 , that there exists a contractive projection on $A_{*}$ with range the norm-closed linear span ${\overline{\operatorname{lin}\left\{x_{j}: j \in \Lambda\right\}}}^{n}$ of the family $\left\{x_{j}: j \in \Lambda\right\}$. Proceeding as in the proof of Corollary 4.2, define the projections $\left\{R_{j}: j \in \Lambda\right\}$ on $A_{*}$, for an element $z$ in $A_{*}$, by

$$
R_{j} z=z\left(u_{j}\right) x_{j}
$$

As in the proof of $\left[27\right.$, Theorem 5.4], the net $\left(\sum_{j \in \lambda_{0}} R_{j}\right)_{\Lambda_{0} \in \Gamma}$, where $\Gamma$ is the set of finite subsets of $\Lambda$, converges in the strong operator topology to a contractive projection $R$ on $A_{*}$, the adjoint $R^{*}$ of which is the required weak*-continuous contractive projection with range $\varlimsup_{\operatorname{lin}\left\{u_{j}: j \in \Lambda\right\}} w^{*}$.

Acknowledgements. The authors wish to express their gratitude to the late G. T. Rüttimann who made substantial contributions to the research reported in this paper and to the Irish Research Council for Science, Engineering, and Technology for the support that their research has received under grant number R.9854.

\section{References}

[1] T. J. Barton, T. Dang, and G. Horn, Normal representations of Banach Jordan triple systems, Proc. Amer. Math. Soc. 102 (1988), no. 3, 551-555.

[2] T. Barton and R. M. Timoney, Weak*-continuity of Jordan triple products and its applications, Math. Scand. 59 (1986), no. 2, 177-191.

[3] F. F. Bonsall and J. Duncan, Numerical ranges of operators on normed spaces and of elements of normed algebras, London Mathematical Society Lecture Note Series, vol. 2, Cambridge University Press, London, 1971. 
[4] L. J. Bunce and C.-H. Chu, Compact operations, multipliers and Radon-Nikodým property in JB*-triples, Pacific J. Math. 153 (1992), no. 2, 249-265.

[5] M. D. Choi and E. G. Effros, Injectivity and operator spaces, J. Funct. Anal. 24 (1977), no. 2, 156-209.

[6] S. Dineen, Complete holomorphic vector fields on the second dual of a Banach space, Math. Scand. 59 (1986), no. 1, 131-142.

[7] - The second dual of a JB* triple system, Complex Analysis, Functional Analysis and Approximation Theory (Campinas, 1984), North-Holland Math. Stud., vol. 125, North-Holland, Amsterdam, 1986, pp. 67-69.

[8] C. M. Edwards, On Jordan $W^{*}$-algebras, Bull. Sci. Math. (2) 104 (1980), no. 4, 393-403.

[9] _ Rigidly collinear pairs of structural projections on a $\mathrm{JBW}^{*}$-triple, Acta Sci. Math. (Szeged) 72 (2006), no. 1-2, 205-235.

[10] C. M. Edwards, R. V. Hügli, and G. T. Rüttimann, A geometric characterization of structural projections on a JBW*-triple, J. Funct. Anal. 202 (2003), no. 1, 174-194.

[11] C. M. Edwards, D. Lörch, and G. T. Rüttimann, Compatible subtriples of Jordan*-triples, J. Algebra 216 (1999), no. 2, 707-740.

[12] C. M. Edwards, K. McCrimmon, and G. T. Rüttimann, The range of a structural projection, J. Funct. Anal. 139 (1996), no. 1, 196-224.

[13] C. M. Edwards and G. T. Rüttimann, On the facial structure of the unit balls in a JBW*-triple and its predual, J. London Math. Soc. (2) 38 (1988), no. 2, 317-332.

[14] _ A characterization of inner ideals in JB*-triples, Proc. Amer. Math. Soc. 116 (1992), no. 4, 1049-1057.

[15] _ Structural projections on JBW*-triples, J. London Math. Soc. (2) 53 (1996), no. 2, 354-368.

[16] _ _ Peirce inner ideals in Jordan*-triples, J. Algebra 180 (1996), no. 1, 41-66.

[17] _ Involutive and Peirce gradings in JBW*-triples, Comm. Algebra 31 (2003), no. 6, $2819-2848$.

[18] E. G. Effros and E. Størmer, Positive projections and Jordan structure in operator algebras, Math. Scand. 45 (1979), no. 1, 127-138.

[19] Y. Friedman, Bounded symmetric domains and the $\mathrm{JB}^{*}$-triple structure in physics, Jordan algebras (Oberwolfach, 1992), de Gruyter, Berlin, 1994, pp. 61-82.

[20] _ Physical applications of homogeneous balls, Progress in Mathematical Physics, vol. 40, Birkhäuser Boston Inc., Boston, MA, 2005.

[21] Y. Friedman and Y. Gofman, Why does the geometric product simplify the equations of physics? Internat. J. Theoret. Phys. 41 (2002), no. 10, 1841-1855.

$[22] \_$, Relativistic linear spacetime transformations based on symmetry, Found. Phys. 32 (2002), no. 11, 1717-1736.

[23] Y. Friedman and B. Russo, Structure of the predual of a JBW*-triple, J. Reine Angew. Math. 356 (1985), 67-89.

[24] H. Hanche-Olsen and E. Størmer, Jordan operator algebras, Pitman, London, 1984.

[25] G. Horn, Characterization of the predual and ideal structure of a $\mathrm{JBW}^{*}$-triple, Math. Scand. 61 (1987), no. 1, 117-133.

[26] R. V. Hügli, Structural projections on a JBW*-triple and GL-projections on its predual, J. Korean Math. Soc. 41 (2004), no. 1, 107-130.

$[27] \_$, Collinear systems and normal contractive projections in JBW*-triples (2006), preprint. 
[28] W. Kaup, A Riemann mapping theorem for bounded symmetric domains in complex Banach spaces, Math. Z. 183 (1983), no. 4, 503-529.

[29] _ Contractive projections on Jordan $C^{*}$-algebras and generalizations, Math. Scand. 54 (1984), no. 1, 95-100.

[30] O. Loos, Jordan pairs, Lecture Notes in Mathematics, vol. 460, Springer-Verlag, Berlin, 1975.

[31] _ On the socle of a Jordan pair, Collect. Math. 40 (1989), no. 2, 109-125 (1990).

[32] K. McCrimmon, Compatible Peirce decompositions of Jordan triple systems, Pacific J. Math. 103 (1982), no. $1,57-102$.

[33] _ _ A taste of Jordan algebras, Universitext, Springer-Verlag, New York, 2004.

[34] K. McCrimmon and K. Meyberg, Coordinatization of Jordan triple systems, Comm. Algebra 9 (1981), no. 14, 1495-1542.

[35] M. Neal and B. Russo, Contractive projections and operator spaces, Trans. Amer. Math. Soc. 355 (2003), no. 6, 2223-2262 (electronic).

[36] E. Neher, Involutive gradings of Jordan structures, Comm. Algebra 9 (1981), no. 6, 575-599.

[37] _ Jordan triple systems by the grid approach, Lecture Notes in Mathematics, vol. 1280, Springer-Verlag, Berlin, 1987.

[38] G. K. Pedersen, $C^{*}$-algebras and their automorphism groups, London Mathematical Society Monographs, vol. 14, Academic Press Inc., London, 1979.

[39] S. Sakai, $C^{*}$-algebras and $W^{*}$-algebras, Vol. 60, Springer-Verlag, New York, 1971. Ergebnisse der Mathematik und ihrer Grenzgebiete.

[40] L. L. Stachó, A projection principle concerning biholomorphic automorphisms, Acta Sci. Math. (Szeged) 44 (1982), no. 1-2, 99-124.

[41] H. Upmeier, Symmetric Banach manifolds and Jordan $C^{*}$-algebras, North-Holland Mathematics Studies, vol. 104, North-Holland Publishing Co., Amsterdam, 1985.

[42] _ Jordan algebras in analysis, operator theory, and quantum mechanics, CBMS Regional Conference Series in Mathematics, vol. 67, American Mathematical Society, Providence, 1987.

[43] J. D. M. Wright, Jordan $C^{*}$-algebras, Michigan Math. J. 24 (1977), no. 3, 291-302.

[44] M. A. Youngson, A Vidav theorem for Banach Jordan algebras, Math. Proc. Cambridge Philos. Soc. 84 (1978), no. 2, 263-272. 